\title{
X-ray Crystal Structures of a Benzonorbornenyl \\ Cation and of a Protonated Benzonorbornenol
}

\author{
Thomas Laube
}

Cilag AG, Hochstrasse 201-209, CH-8205 Schaffhausen, Switzerland

E-mail: thaube@cilch.jnj.com

\section{List of contents}

Table S1 (Data of the measured crystals)

Table S2 (Selected geometrical data of Me-1 $\mathbf{1}^{+}, 5^{+}, \mathbf{6 , 1 8}$ )

Experimental Section

Crystal structure analysis of $\mathrm{Me}^{+} \mathbf{1}^{+} \mathrm{Sb}_{2} \mathrm{~F}_{11}{ }^{-}$

Some photos showing the preparation of $\mathrm{Me}-1^{+} \mathrm{Sb}_{2} \mathrm{~F}_{11}{ }^{-}$

Crystal structure analysis of $5^{+} \mathrm{SO}_{3} \mathrm{Cl}^{-} \cdot \mathrm{CH}_{2} \mathrm{Cl}_{2}$

Thermal stability of crystalline $5^{+} \mathrm{SO}_{3} \mathrm{Cl}^{-} \cdot \mathrm{CH}_{2} \mathrm{Cl}_{2}$

$5^{+} \mathrm{SO}_{3} \mathrm{Cl}^{-}$or $18 \cdot \mathrm{ClSO}_{3} \mathrm{H}$ (crystal geometry; DFT) $\quad \mathrm{S} 73$

$\mathbf{5}^{+}, 18$ (semiemp.) $\quad \mathrm{S77}$

$\mathbf{5}^{+}$(in water; DFT) $\quad S 80$

6 (DFT)

$\mathbf{1 8} \cdot \mathrm{ClSO}_{3} \mathrm{H}(\mathrm{DFT}) \quad \mathrm{S} 87$

$\mathrm{SO}_{3} \mathrm{Cl}^{-}$(DFT) $\quad \mathrm{S} 90$

$\mathrm{Sb}_{2} \mathrm{~F}_{11}{ }^{-}$(DFT; incl. Cambridge File results) $\quad \mathrm{S} 92$

Neutral model for a classical isomer of $\mathrm{Me}^{+\mathbf{1}^{+}} \quad \mathrm{S} 101$

Additional data about related structures reported in the literature $\quad$ S104 
: (c) 2004 American Chemical Society, J. Am. Chem. Soc., Laube ja040115t Supporting Info Page 2 Thomas Laube J. Am. Chem. Soc.

Table S1. Data of the measured crystals.

\begin{tabular}{|c|c|c|}
\hline & $\mathrm{Me}^{-1^{+}} \mathrm{Sb}_{2} \mathrm{~F}_{11}^{-}$ & $5^{+} \mathrm{SO}_{3} \mathrm{Cl}^{-} \cdot \mathrm{CH}_{2} \mathrm{Cl}_{2}$ \\
\hline formula & $\mathrm{C}_{12} \mathrm{H}_{13} \mathrm{~F}_{11} \mathrm{Sb}_{2}$ & $\mathrm{C}_{12} \mathrm{H}_{15} \mathrm{Cl}_{3} \mathrm{O}_{4} \mathrm{~S}$ \\
\hline formula weight & 609.72 & 361.65 \\
\hline crystal system & monoclinic & monoclinic \\
\hline$Z$ (formula) & 2 & 4 \\
\hline$Z^{\prime}$ & $1 / 2$ & 1 \\
\hline space group & $P 2_{1} / m$ & $P 2_{1} / n$ \\
\hline$a / \AA$ & $6.7320(2)$ & $10.97570(10)$ \\
\hline$b / \AA$ & $9.2033(2)$ & $6.17250(10)$ \\
\hline$c / \AA ̊$ & $14.4010(5)$ & $23.3650(3)$ \\
\hline$\alpha / \operatorname{deg}$ & 90 & 90 \\
\hline$\beta / \operatorname{deg}$ & $99.6800(14)$ & $103.3466(5)$ \\
\hline$\gamma / \operatorname{deg}$ & 90 & 90 \\
\hline$V / \AA^{3}$ & $879.53(4)$ & $1540.17(3)$ \\
\hline Temperature / K & $153(2)$ & $160(2)$ \\
\hline$R 1\left(I>2 \sigma_{I}\right)$ & 0.0391 & 0.0392 \\
\hline$w R 2\left(I>2 \sigma_{I}\right)$ & 0.0802 & 0.1004 \\
\hline g. o. f. & 1.103 & 1.025 \\
\hline
\end{tabular}




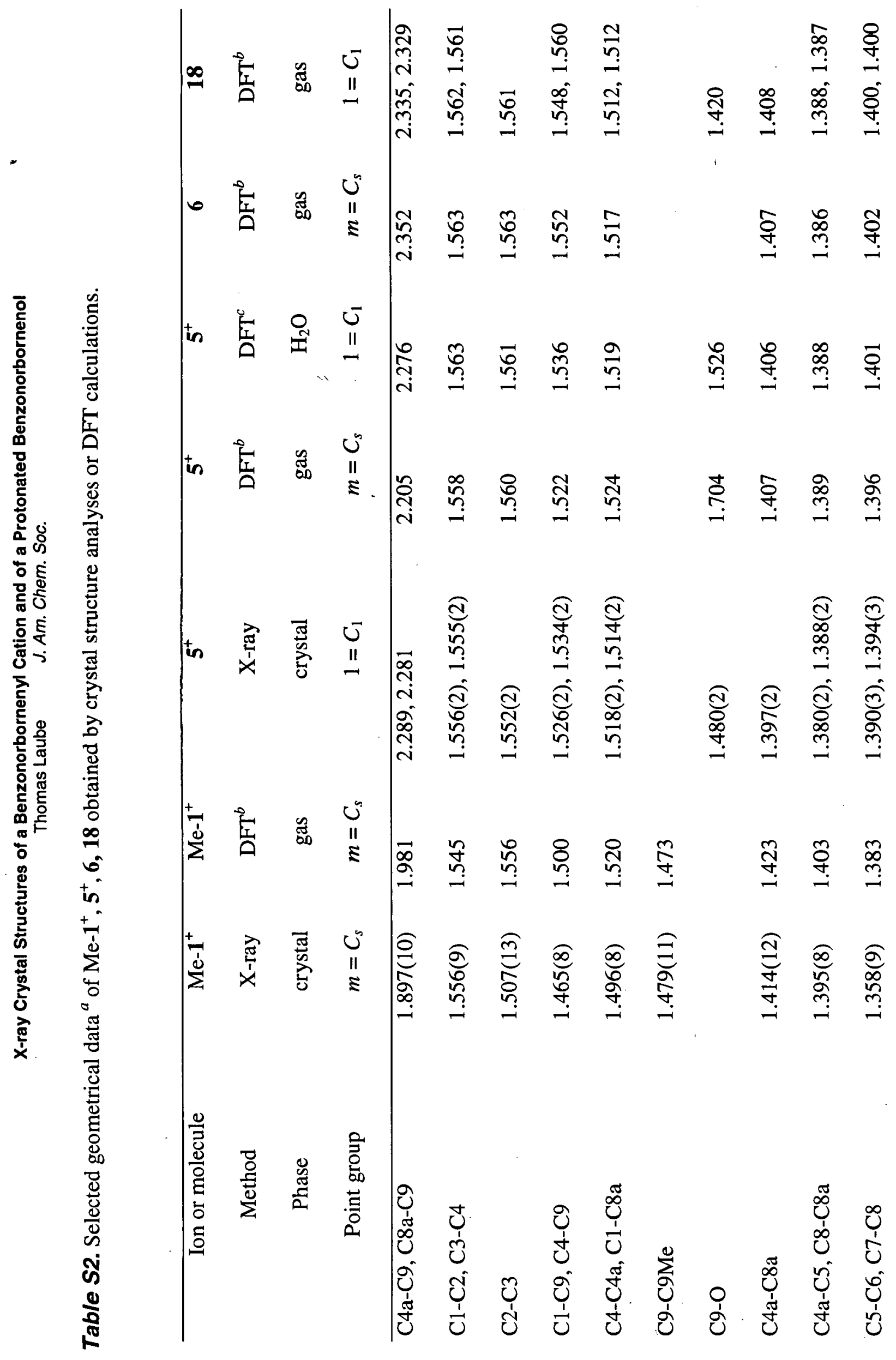




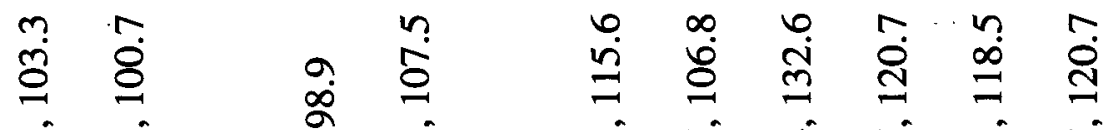

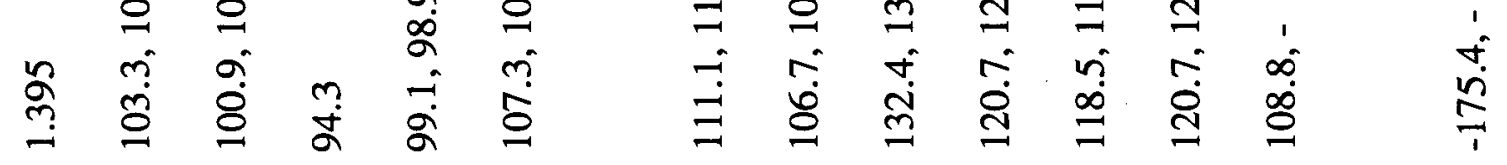

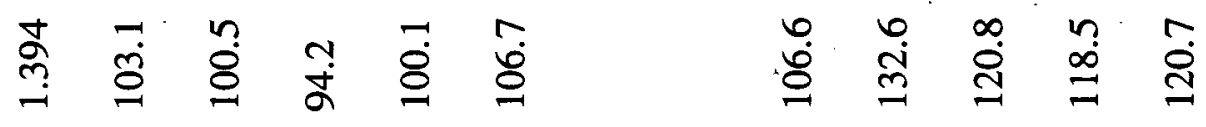

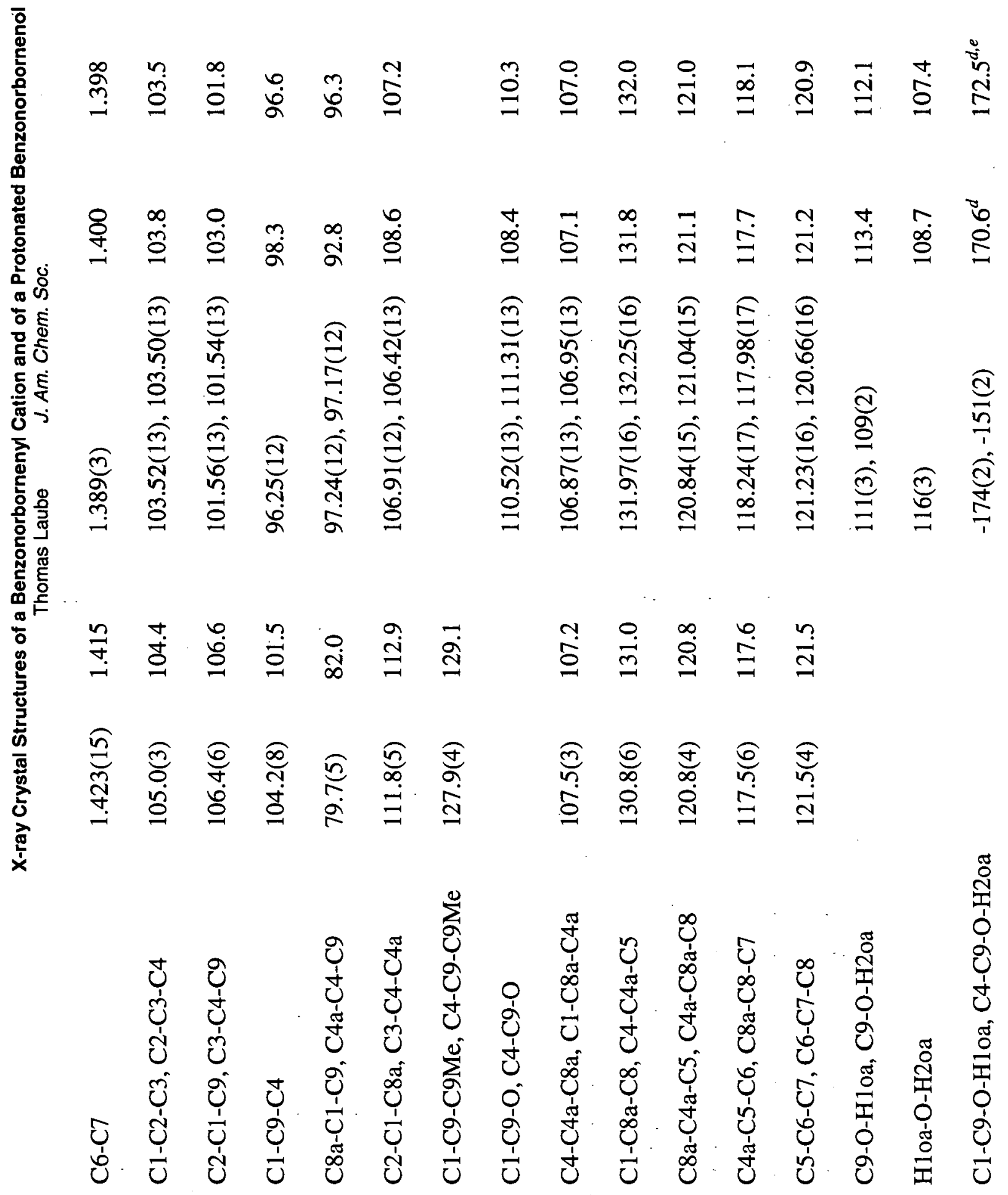




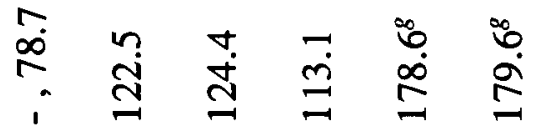

$$
\begin{aligned}
& \text { 言总 } \cong
\end{aligned}
$$

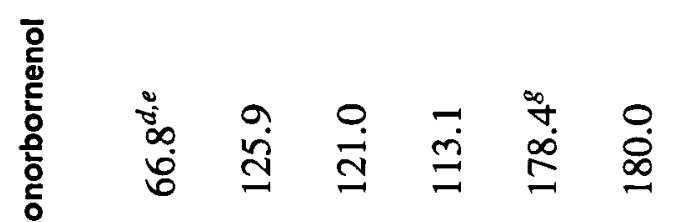

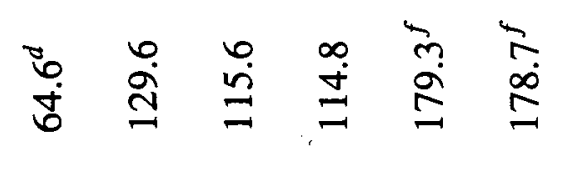

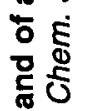

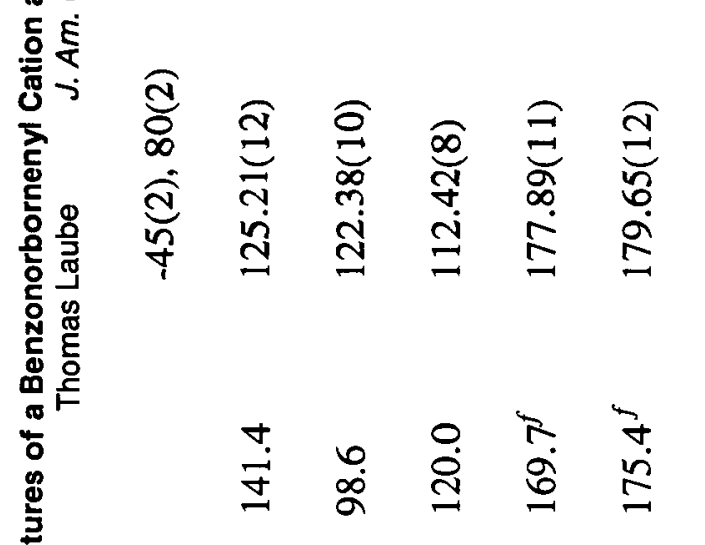

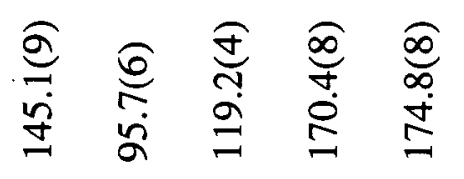

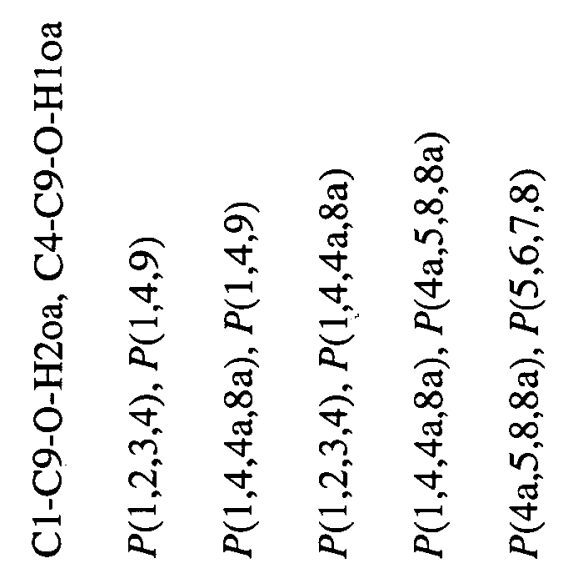

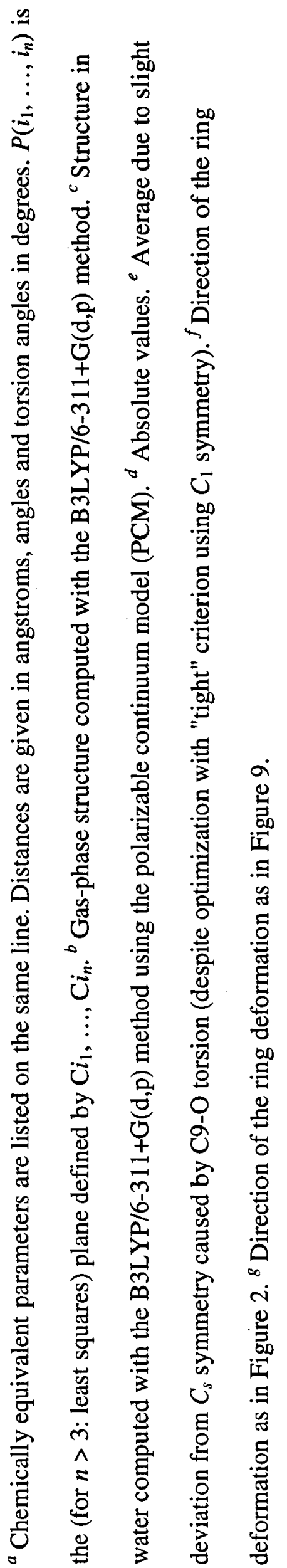


(1) 2004 American Chemical Society, J. Am. Chem. Soc.. Laube ja040115t Supporting Info Page 6 Thomas Laube J. Am. Chem. Soc.

\section{Experimental Section}

General Remarks. Flash chromatography has been carried out using silica gel 60 (particle size $0.04-0.063 \mathrm{~mm}$; Fluka). The solvents for the experiments with the cation salts were refluxed over $\mathrm{P}_{2} \mathrm{O}_{5}$ under nitrogen, distilled, and stored in Aldrich Sure/Stor flasks or Aldrich storage bottles without joints. All cation experiments were carried out under dry nitrogen. The crystals were selected at about $-70 \mathrm{deg} \mathrm{C}$ and mounted on glass needles as described in ref. 16 at Cilag AG and transported in a nitrogen atmosphere at about $-120 \mathrm{deg} C$ to the University of Zurich. The single crystal diffraction measurements were carried out with a Nonius FR590 sealed tube $3 \mathrm{~kW}$ generator and a Nonius KappaCCD four circle goniometer with a CCD area detector using $\mathrm{MoK}_{\alpha}$ radiation. An Oxford Cryostream 700 low temperature device was used for crystal cooling. The data reductions were carried out with teXsan, ${ }^{57}$ the absorption corrections (based on multi-scans from symmetry-related measurements) were carried out with Sortav. ${ }^{58}$

\section{1,2,3,4-Tetrachloro-1,4-dihydro-9,9-dimethoxy-1,4-methanonaphthalene (12). It is} recommended to wear a face shield and protective gloves during the handling of crystalline 10. The preparation of $\mathbf{1 0}$ starting with $27.7 \mathrm{~g}$ of 9 was carried out in a 1 L PTFE beaker with a PVDF cover according to ref. 11. The precipitated diazonium salt was isolated using a PTFE spatula, PP Buchner funnel, and a PP filtering flask. The wet $10(38.5-42.5 \mathrm{~g})$ was reacted with $80 \mathrm{~g}$ of $\mathbf{8}$ as described in ref. 12. The volatile components were removed with a rotary evaporator at a bath temperature of $70 \mathrm{deg} \mathrm{C}$. The raw product was purified by flash chromatography (cyclohexane:toluene, 80:20, v:v; $1.3 \mathrm{~kg}$ of silica gel; the product crystallizes in the receiver tubes). The combined product charges of four such reaction sequences (scaling up is not recommended for safety reasons) were recrystallized from cyclohexane yielding 80.3 $\mathrm{g}$ of 12. 
(C) 2004 American Chemical Society, J. Am. Chem. Soc.: Laube ja040115t Supporting Info Page 7 Thomas Laube J. Am. Chem. Soc.

1,2,3,4-Tetrahydro-9,9-dimethoxy-1,4-methanonaphthalene (13). The dehalogenation was carried out two times starting with each $40 \mathrm{~g}$ of 12 according to ref. 13. Kugelrohr distillation yielded 21.8 and $23.5 \mathrm{~g}$ of raw product mixture (13 and 13a). The combined raw products were hydrogenated ${ }^{13}$ and purified by flash chromatography (cyclohexane/ethyl acetate, $90: 10, \mathrm{v}: \mathrm{v})$ to give $33.9 \mathrm{~g}$ of 13 .

1,2,3,4-Tetrahydro-1,4-methanonaphthalen-9-one (14). The hydrolysis of $33.9 \mathrm{~g}$ of 13 was carried out according to ref. 14 . The product was purified by flash chromatography with ether and subsequent Kugelrohr distillation ( $130 \mathrm{deg}$ C/ 0.003 Torr; receiver cooled to -60 $\operatorname{deg} \mathrm{C}$ ) to yield $25.7 \mathrm{~g}$ of $\mathbf{1 4}$ (low-melting oil, stored in a freezer).

1,2,3,4-Tetrahydro-9-methyl-1,4-methanonaphthalene-9-anti-ol (15) was prepared according to ref. $4 \mathrm{a}$ by reaction of $6.32 \mathrm{~g}$ of 14 with $\mathrm{MeMgI}$ in ether and subsequent flash chromatography (cyclohexane/ethyl acetate, 80:20, v:v) to give $6.3 \mathrm{~g}$ of 15 .

9-Chloro-9-methyl-1,2,3,4-tetrahydro-1,4-methanonaphthalene (16). In a $500 \mathrm{~mL}$ round-bottom flask, $0.95 \mathrm{~g}$ of 15 in $50 \mathrm{~mL}$ of pentane were reacted with $250 \mathrm{~mL}$ of conc. $\mathrm{HCl}$ for about $5.5 \mathrm{~h}$ at room temperature (reaction control by TLC using cyclohexane/ethyl acetate, $80: 20, v / v)$. After phase separation, the aqueous phase was washed three times each with 50 $\mathrm{mL}$ of pentane, the combined organic phases were washed with conc. $\mathrm{NaHCO}_{3}$ solution, water, conc. $\mathrm{NaCl}$ solution, and then dried over $\mathrm{K}_{2} \mathrm{CO}_{3}$. After removal of the solvent, the raw product was distilled under vacuum in a Kugelrohr apparatus (receiver flask cooled to -80 $\operatorname{deg} \mathrm{C}$ ) to give $0.87 \mathrm{~g}$ of a colourless oil that solidifies in a freezer.

\section{9-Fluoro-9-methyl-1,2,3,4-tetrahydro-1,4-methanonaphthalene (17). A $100 \mathrm{~mL}$ FEP}

bottle with a hole in the screw closure was charged with $10 \mathrm{~mL}$ of pentane and $25 \mathrm{~mL}$ of $\mathrm{HF} /$ pyridine (Fluka). Under stirring at $0 \mathrm{deg} \mathrm{C}$, a suspension of $2.20 \mathrm{~g}$ of $15 \mathrm{in} 60 \mathrm{~mL}$ of pentane was added with a syringe with a thick PFA needle within about $10 \mathrm{~min}$. After $45 \mathrm{~min}$ both phases were clear (TLC control as described for 16), and the reaction mixture was poured into ice/water in a FEP separatory funnel. The organic phase was quickly washed with 
(C) 2004 American Chemical Society, J. Am. Chem. Soc., Laube ja040115t Supporting Info Page 8 Thomas Laube J. Am. Chem. Soc.

half-conc. $\mathrm{NaHCO}_{3}$ solution and then with water and dried over $\mathrm{Na}_{2} \mathrm{SO}_{4}$ at 0 deg C. After filtration, most of the solvent was removed with a rotary evaporator at room temperature, and then the raw product was distilled under vacuum in a Kugelrohr apparatus (receiver flask cooled to $-80 \mathrm{deg}$ C) to give $1.77 \mathrm{~g}$ of a colourless distillate, which was stored in a freezer.

1,2,3,4-Tetrahydro-1,4-methanonaphthalene-9-anti-ol (18) was prepared in two batches from totally $7.99 \mathrm{~g}$ of 14 and $49.45 \mathrm{~g}$ of $\mathrm{LiAlH}(\mathrm{O} t \mathrm{Bu})_{3}$ (Fluka) as described in ref. 17. The pure anti isomer (18) was separated from a small amount of the syn isomer by flash chromatography (column diameter $5 \mathrm{~cm}$, column length $1 \mathrm{~m}$ ) using cyclohexane/ethyl acetate (70:30, v:v), which yielded $6.61 \mathrm{~g}$ of $\mathbf{1 8}$ as colourless crystals.

(deloc-4a,8a,9)-1,2,3,4-Tetrahydro-9-methyl-1,4-methanonaphthalen-9-ylium undecafluorodiantimonate $\left(\mathbf{M e - 1}{ }^{+} \mathbf{S b}_{2} \mathbf{F}_{11}\right)$. The reaction was carried out using the apparatus shown in figure 6 of ref. 16 . A solution of $0.34 \mathrm{~g}$ of $17(1.93 \mathrm{mmol})$ in $7 \mathrm{~mL}$ of $\mathrm{CCl}_{2} \mathrm{FCClF}_{2}$ was added with a syringe to $45 \mathrm{~mL}$ of $\mathrm{CFCl}_{3}$ in compartment $\mathrm{A}$ of the reaction vessel (rubber septum on joint 3 ) at about -90 deg C. The septum on joint 3 was replaced by a $10 \mathrm{~mL}$ PTFE dropping funnel, and a freshly prepared solution of $1.07 \mathrm{~g}$ of SbF $5(4.94 \mathrm{mmol}$; Merck) in $9 \mathrm{~mL}$ of $\mathrm{CCl}_{2} \mathrm{FCClF}_{2}$ was added with the dropping funnel within $8 \mathrm{~min}$. to the solution in A (bath temperature: -88 to $-86 \mathrm{deg} \mathrm{C}$ ). The yellow, slightly turbid reaction mixture slowly turns orange-brown. After keeping the reaction mixture at about $-85 \mathrm{deg} \mathrm{C}$ for ca. 40 min., the dropping funnel is replaced by a stopcock adapter, and the mixture is filtered into B by applying nitrogen pressure from the stopcock on joint 3 and vacuum through stopcock 1 . The filtration at about $-90 \mathrm{deg}$. C takes ca. $50 \mathrm{~min}$. (this value varies strongly from experiment to experiment). The mother liquor in $\mathrm{A}$ is removed with a long needle through stopcock 1, and the glassy, brownish filter cake on the frit is dried during $90 \mathrm{~min}$. by applying vacuum ( $\mathrm{p}<0.01$ Torr) at stopcock 1 and the adapter on joint 3 and by slow raising of the bath temperature from -85 to -55 deg $\mathrm{C}$. The filter cake turns yellow-brown on drying. After cooling the bath to $-90 \mathrm{deg} \mathrm{C}$ and filling the apparatus with nitrogen, the filter cake is 
(1) 2004 American Chemical Society, J. Am. Chem. Soc., Laube ja040115t Supporting Info Page 9 Thomas Laube J. Am. Chem. Soc.

coarsely powdered with a long spatula through joint 3 (flushed with nitrogen). The whole apparatus is quickly turned left by ca. 100 degrees so that the precooled filtration vessel could be cooled to ca. -80 deg $\mathrm{C}$ in a hemispherical Dewar vessel, and the raw powdered salt was transferred as quickly as possible with the spatula from $\mathrm{A}$ into $\mathrm{C}$. The reaction vessel on joint 5 is replaced by an adapter with a three-way stopcock with a septum and a nitrogen inlet. The salt was cooled to about $-105 \operatorname{deg} \mathrm{C}$ (filtration apparatus in position I), and $20 \mathrm{~mL}$ of $\mathrm{CH}_{2} \mathrm{Cl}_{2}$ was slowly added with a syringe through the septum on the three-way stopcock on joint 5 . Nevertheless the liquid phase turns dark red, and most of the raw salt turns into a sticky brown material during this process, probably due to decomposition. The bath temperature is raised to $-80 \mathrm{deg} \mathrm{C}$ to improve the solubility, and after stirring for about $10 \mathrm{~min}$., the mixture is filtered into $\mathrm{D}$ by turning the filtration apparatus into position II. A large amount of the tarlike material remained on the frit. The clear yellow filtrate is poured into the precooled $50 \mathrm{~mL}$ two-neck flask with a three-way stopcock adapter on joint 6 . The flask is then disconnected from joint 6, and equipped with an additional wide-bore stopcock adapter. The flask is placed into a 3-L Dewar with a dry ice-ethanol mixture, connected to a cold trap, and vacuum (ca. 0.002 Torr) is applied for about $18 \mathrm{~h}$. One obtains a brown viscous film, which contains some small crystals in the upper part indicating that crystal growth probably took place only at the beginning of the evaporation of the solvent. The microscopic investigation and the mounting were carried out as described in ref. 16 . The thermal stability of the crystals has been determined by stepwise warming up of crystal samples covered with perfluorinated polyether oil on the cold stage in separate runs. The crystals decompose around $-30^{\circ} \mathrm{C}$.

\section{1,2,3,4-Tetrahydro-1,4-methanonaphthalenyl-9-anti-oxonium chlorosulfonate •}

methylene chloride $\left(5^{+} \mathrm{SO}_{3} \mathrm{Cl}^{-} \cdot \mathrm{CH}_{2} \mathrm{Cl}_{2}\right)$. In a $50 \mathrm{~mL}$ two neck flask with a three-way stopcock with a serum stopper and a nitrogen inlet and a connection adapter to a high-vacuum system, $20 \mathrm{~mL}$ of methylene chloride were stirred at ca. $-80 \mathrm{deg} \mathrm{C}$, and $210 \mu \mathrm{L}$ of $\mathrm{ClSO}_{3} \mathrm{H}$ (ca. $0.37 \mathrm{~g} ; 3.2 \mathrm{mmol}$; Fluka) were added slowly with a gas-tight $500 \mu \mathrm{L}$ glass syringe 
-(C) 2004 American Chemical Society, J. Am. Chem. Soc., Laube ja040115t Supporting Info Page 10 Thomas Laube J. Am. Chem. Soc.

(Hamilton \# 1750) equipped with a PTFE needle. A solution of $0.51 \mathrm{~g}$ of $\mathbf{1 8}(3.2 \mathrm{mmol})$ in 5 $\mathrm{mL}$ of methylene chloride was added within $5 \mathrm{~min}$. High vacuum was applied slowly to the clear solution under stirring at ca. $-80 \mathrm{deg} C$, then the stirrer was switched off. After pumping off the solvent for about $24 \mathrm{~h}$, a viscous solution without crystals has been formed. The flask was filled with nitrogen and kept at ca. $-80 \mathrm{deg}$ C. Within one day the crystallization yielded intergrown crusts consisting mainly of crystals. A small amount of mother liquor was removed with a syringe, and the crystals were dried at $-80 \operatorname{deg} \mathrm{C}$ for $4 \mathrm{~h}$. The microscopic investigation and the mounting were carried out as described in ref. 16. In a separate run, the temperature of the cold stage of the microscope was raised linearly in time from -70 deg $\mathrm{C}$ to $+10 \mathrm{deg} \mathrm{C}$ within $8 \mathrm{~h}$, and a photo was made every $5 \mathrm{~min}$ to follow the decomposition. The crystals start decomposing or melting around $-40^{\circ} \mathrm{C}$.

Crystal Structure Analysis of $\mathrm{Me}-\mathbf{1}^{+} \mathrm{Sb}_{2} \mathrm{~F}_{11}{ }^{-}$. The small yellow crystal was difficult to locate in the drop of frozen oil so that its size could only be guessed $(0.2 \times 0.1 \times 0.05 \mathrm{~mm})$. The crystal was measured at $-120 \mathrm{deg} C$ with a monoclinic cell (1648 unique reflections up to $\left.\theta=25^{\circ}\right)$ and initially solved $\left(\right.$ SHELXS $\left.{ }^{59}\right)$ and refined $\left(\right.$ SHELXL $\left.^{60}\right)$ in $P 2_{1} / n$ with four ion pairs in the unit cell. Undistorted pictures of some planes in the reciprocal space generated from the measured frames showed some rings indicating the presence of a small amount of powdery material. Analysis of the refined structure with PLATON ${ }^{61}$ and of the reciprocal space data with FOTOPLOT ${ }^{62}$ indicated that the cell could be reduced and the space group changed to $P 2{ }_{1} / m$ with two ion pairs in the unit cell (cation and anion with crystallographic $m$ symmetry, $Z^{\prime}=1 / 2$ ). Large ADPs of most of the fluorine atoms indicated rotational disorder of the $\mathrm{Sb}_{2} \mathrm{~F}_{11}{ }^{-}$anion along its $\mathrm{Sb}$-Sb axis. The result of a search in the Cambridge Structural Database ${ }^{63}$ for the geometry and the conformation distribution of $\mathrm{Sb}_{2} \mathrm{~F}_{11}{ }^{-}$anions was used as basis for the construction of a threefold disorder model of the anion using reasonable conformations with SYBYL 6.0. ${ }^{64}$ The three sites have common Sb positions. One site has $m$ 
$=C_{s}$ symmetry, and its fluorine atoms have the names F $x \mathrm{a}$ or Fxa' where $x$ is a number and the prime indicates a mirror symmetry-related atom. The second site has $1=C_{1}$ symmetry and fluorine atom names Fxb, and the third site is related to the second site by the crystallographic mirror plane and thus has fluorine atom names Fxb'. The sum of the three site occupation factors is equal to 1 . Several geometrical constraints (DFIX) in the anion sites were introduced to keep the refinement stable: each anion was treated as if it consists of two rigid pseudo-octahedra having only $4 m m=C_{4 v}$ symmetry with a common corner (the bridging fluorine atom F1 $y$ which lies on both fourfold axes; $\left.y=\mathrm{a}, \mathrm{b}, \mathrm{b}^{\prime}\right)$. This construction allows for each anion only three internal degrees of freedom: two torsion angles around the Sb1-F1y and $\mathrm{Sb2-F1y} \mathrm{bonds} \mathrm{for} \mathrm{the} \mathrm{mutual} \mathrm{orientation} \mathrm{of} \mathrm{the} \mathrm{pseudo-octahedra,} \mathrm{and} \mathrm{the} \mathrm{angle} \mathrm{Sb1-F1y-}$ Sb2. In addition, all anions had a common variable size scaling factor. The ADPs of all $F$ atoms were forced to approximate isotropic behavior using ISOR restraints, and F1 and F11a were forced to have the same ADPs with EADP. This model represents essentially two deformed and irregular rings of electron density around the $\mathrm{Sb}$ atoms which upon refinement explains the experimental data well. It must be emphasized that other disorder models with even more disordered sites or other constraints could perhaps replace our disorder model. No constraints were applied to the carbon atoms of the cation which lies on the mirror plane, but its hydrogen atoms were refined with the constraints AFIX 13 (H1), AFIX 23 (H2n, H2x), AFIX $43(\mathrm{H} 7, \mathrm{H} 8)$, and AFIX 133 (methyl hydrogens) and $U_{\text {iso }}(\mathrm{H})=1.2 U_{\mathrm{eq}}(\mathrm{C})$ in all cases. The refinement leads finally to $R 1=0.0391, w R 2=0.0802$. The estimated relative energies of the anion with $F$ names $F x a$ or $F x a$ ' is 0.1 , that of the anions with $F$ names $F x b$ or $F x b$ ' is 0.7 $\mathrm{kcal} / \mathrm{mole}$ with respect to the lowest energy conformation of the isolated anion (interpolation based on B3LYP/LANL2DZ/B3LYP/LANL2DZ potential energy surface).

Crystal Structure Analysis of $5^{+} \mathbf{S O}_{3} \mathbf{C l}^{-} \cdot \mathbf{C H}_{2} \mathbf{C l}_{2}$. The colourless crystal was measured at $-113 \mathrm{deg} \mathrm{C}$, its average size may have been around $0.3 \mathrm{~mm}$ but could not be determined in the frozen drop of oil. The structure was solved $\left(\mathrm{SHELXS}^{59}\right)$ and refined $\left(\mathrm{SHELXL}^{60}\right)$ in 
(C) 2004 American Chemical Society, J. Am. Chem. Soc., Laube ja040115t Supporting Info Page 12 Thomas Laube J. Am. Chem. Soc.

$P 2_{1} / n$ with four ion pairs in the unit cell. All positional and thermal parameters of the hydrogen atoms were refined without constraints. The $U_{\text {iso }}$ values of hydrogen atoms on the oxygen atom were larger than those of the other $\mathrm{H}$ atom of the cation. Although the $\mathrm{C}-\mathrm{O}$ bond is the only rotatable bond in the cation, the short $\mathrm{H}^{\cdots} \mathrm{O}_{\text {anion }}$ distances indicate strong hydrogen bonds, which should raise the energy barrier for the rotation around the $\mathrm{C}-\mathrm{O}$ bond. Therefore the larger $U_{\text {iso }}$ values of these $\mathrm{H}$ atoms were interpreted as hints to site occupation factors less than 1 . The refinement without positional and thermal constraints yielded site occupation factors around 0.7 for the $\mathrm{H}$ atoms on $\mathrm{O}(\mathrm{H} 1 \mathrm{oa}, \mathrm{H} 2 \mathrm{oa})$, where those of the other $\mathrm{H}$ atoms of the cation remained close to 1 . The residual $\mathrm{H}$ populations ( $\mathrm{H} 1 \mathrm{ob}, \mathrm{H} 2 \mathrm{ob}$; close to the nearest $\mathrm{O}_{\text {anion }}$ atoms $\mathrm{O} 1$ and $\left.\mathrm{O} 2\right)$ were refined with AFIX 147 constraints, $U_{\text {iso }}(\mathrm{H} 1 \mathrm{oa})=U_{\text {iso }}(\mathrm{Hlob})$, $U_{\text {iso }}(\mathrm{H} 2 \mathrm{oa})=U_{\text {iso }}(\mathrm{H} 2 \mathrm{ob})$, and $\operatorname{sof}(\mathrm{H} 1 \mathrm{oa})+\operatorname{sof}(\mathrm{H} 1 \mathrm{ob})=1, \operatorname{sof}(\mathrm{H} 2 \mathrm{oa})+\operatorname{sof}(\mathrm{H} 2 \mathrm{ob})=1$. The refinement lead finally to $R 1=0.0392, w R 2=0.1004$. 
-(C) 2004 American Chemical Society, J. Am. Chem. Soc., Laube ja040115t Supporting Info Page 13 Thomas Laube J. Am. Chem. Soc.

\section{Crystal structure analysis of $\mathrm{Me}-1^{+} \mathrm{Sb}_{2} \mathrm{~F}_{11}^{-}$}

Table 1. Crystal data and structure refinement for $\mathrm{Me}^{-1} \mathrm{Sb}_{2} \mathrm{~F}_{11}{ }^{-}$.

\begin{tabular}{|c|c|}
\hline Identification code & c10201 \\
\hline Empirical formula & $\mathrm{C} 6 \mathrm{H} 6.50 \mathrm{~F} 5.50 \mathrm{Sb}$ \\
\hline Formula weight & 304.86 \\
\hline Temperature & $153(2) K$ \\
\hline Wavelength & $0.71073 \AA$ \\
\hline Crystal system, space group & monoclinic, $P Z_{1} / m$ \\
\hline Unit cell dimensions & $\begin{array}{ll}a=6.7320(2) \AA & \alpha=90 \mathrm{deg} . \\
b=9.2033(2) \AA & \beta=99.6800(14) \mathrm{deg} . \\
c=14.4010(5) \AA & \gamma=90 \mathrm{deg} .\end{array}$ \\
\hline Volume & $879.53(4) \AA^{3}$ \\
\hline$Z$, Calculated density & 4, $2.302 \mathrm{Mg} / \mathrm{m}^{3}$ \\
\hline Absorption coefficient & $3.173 \mathrm{~mm}^{-1}$ \\
\hline$F(000)$ & 572 \\
\hline Crystal size & $0.20 \times 0.10 \times 0.05 \mathrm{~mm}$ \\
\hline $\begin{array}{l}\text { Theta range for data collection } \\
\text { Limiting indices }\end{array}$ & $\begin{array}{l}3.16 \text { to } 25.01 \mathrm{deg} . \\
-8 \leq h \leq 8,-10 \leq k \leq 8,-17 \leq l \leq 17\end{array}$ \\
\hline Reflections collected / unique & $12218 / 1648[R(\mathrm{int})=0.0770]$ \\
\hline Completeness to theta $=25.01$ & $99.8 \%$ \\
\hline Max. and min. transmission & 0.8575 and 0.5694 \\
\hline Refinement method & Ful1-matrix least-squares on $F$ \\
\hline Data / restraints / parameters & $1648 / 176 / 226$ \\
\hline Goodness-of-fit on $r^{2}$ & 1.103 \\
\hline Final $R$ indices $\left[\begin{array}{ll}I>2 & \sigma_{I}\end{array}\right]$ & $R 1=0.0391, w R 2=0.0802$ \\
\hline$R$ indices (all data) & $R 1=0.0581, \quad w R 2=0.0880$ \\
\hline Extinction coefficient & $0.0000(4)$ \\
\hline Largest diff. peak and hole & 0.739 and -0.456 e $\AA^{-3}$ \\
\hline
\end{tabular}


(C) 2004 American Chemical Society, J. Am. Chem. Soc., Laube ja040115t Supporting Info Page 14 Thomas Laube J. Am. Chem. Soc.

Table 2a. Atomic coordinates and (equivalent) isotropic displacement parameters $\left(\AA^{2}\right)$ for Me-1 ${ }^{+} \mathrm{Sb}_{2} \mathrm{~F}_{11}{ }^{-} . U_{\text {eq }}$ is defined as one third of the trace of the orthogonalized $U_{i j}$ tensor. For $\mathrm{H}$ atoms, $U_{\text {iso }}$ is given. The site occupation factors (sof) should be divided by the symmetry multiplicity $(s m)$ for obtaining the correct content of the asymmetric unit.

\begin{tabular}{|c|c|c|c|c|c|c|}
\hline Atom & $x$ & $y$ & $z$ & $U_{e q}$ or $U_{i s o}$ & sof & $s m$ \\
\hline Sb1 & $0.91570(9)$ & $1 / 4$ & $0.87778(4)$ & $0.0580(2)$ & 1 & 2 \\
\hline $\mathrm{Sb} 2$ & $0.97713(9)$ & $1 / 4$ & $0.61024(4)$ & $0.0564(2)$ & 1 & 2 \\
\hline $\mathrm{F} 1 \mathrm{~A}$ & $0.977(4)$ & $1 / 4$ & $0.7487(9)$ & $0.116(16)$ & $0.28(3)$ & 2 \\
\hline F11A & $0.876(6)$ & $1 / 4$ & $1.0044(14)$ & $0.116(16)$ & $0.28(3)$ & 2 \\
\hline $\mathrm{F} 12 \mathrm{~A}$ & $1.109(3)$ & $0.3921(11)$ & $0.9028(16)$ & $0.16(2)$ & $0.28(3)$ & 1 \\
\hline F13A & $0.724(3)$ & $0.3898(11)$ & $0.8433(19)$ & $0.18(2)$ & $0.28(3)$ & 1 \\
\hline F1B & $0.914(2)$ & $0.2927(14)$ & $0.7396(6)$ & $0.083(6)$ & $0.362(13)$ & 1 \\
\hline F11B & $0.918(2)$ & $0.230(3)$ & $1.0056(7)$ & $0.064(6)$ & $0.362(13)$ & 1 \\
\hline $\mathrm{F} 12 \mathrm{~B}$ & $1.1824(15)$ & $0.2107(17)$ & $0.8852(8)$ & $0.075(8)$ & $0.362(13)$ & 1 \\
\hline F13B & $0.983(3)$ & $0.4496(13)$ & $0.8898(12)$ & $0.090(7)$ & $0.362(13)$ & 1 \\
\hline F14B & $0.6440(16)$ & $0.304(2)$ & $0.8504(10)$ & $0.127(15)$ & $0.362(13)$ & 1 \\
\hline F15B & $0.848(4)$ & $0.0673(16)$ & $0.8454(13)$ & $0.169(13)$ & $0.362(13)$ & 1 \\
\hline F21A & $0.968(4)$ & $1 / 4$ & $0.4794(11)$ & $0.081(17)$ & $0.28(3)$ & 2 \\
\hline F22A & $1.251(2)$ & $1 / 4$ & $0.6383(19)$ & $0.079(11)$ & $0.28(3)$ & 2 \\
\hline F23A & $0.978(2)$ & $0.4489(12)$ & $0.6185(13)$ & $0.099(12)$ & $0.28(3)$ & 1 \\
\hline $\mathrm{F} 24 \mathrm{~A}$ & $0.701(2)$ & $1 / 4$ & $0.600(2)$ & $0.065(10)$ & $0.28(3)$ & 2 \\
\hline F21B & $1.032(3)$ & $0.209(2)$ & $0.4904(9)$ & $0.123(8)$ & $0.362(13)$ & 1 \\
\hline F22B & $1.081(3)$ & $0.0759(17)$ & $0.6627(13)$ & $0.125(9)$ & $0.362(13)$ & 1 \\
\hline F23B & $0.728(2)$ & $0.164(3)$ & $0.5885(10)$ & $0.121(11)$ & $0.362(13)$ & 1 \\
\hline F24B & $0.865(4)$ & $0.4264(18)$ & $0.5782(14)$ & $0.143(12)$ & $0.362(13)$ & 1 \\
\hline F25B & $1.221(2)$ & $0.333(3)$ & $0.6532(13)$ & $0.189(15)$ & $0.362(13)$ & 1 \\
\hline $\mathrm{C} 1$ & $0.6120(10)$ & $0.1244(7)$ & $1.1669(5)$ & $0.0593(16)$ & 1 & 1 \\
\hline $\mathrm{C} 2$ & $0.4719(10)$ & $0.1681(7)$ & $1.0738(5)$ & $0.0662(18)$ & 1 & 1 \\
\hline C7 & $0.4343(9)$ & $0.1727(8)$ & $1.4018(5)$ & $0.0689(19)$ & 1 & 1 \\
\hline $\mathrm{C} 8$ & $0.4887(8)$ & $0.0956(7)$ & $1.3297(5)$ & $0.0584(16)$ & 1 & 1 \\
\hline C8A & $0.5294(9)$ & $0.1732(7)$ & $1.2518(4)$ & $0.0563(15)$ & 1 & 1 \\
\hline C9 & $0.7418(14)$ & $1 / 4$ & $1.1953(6)$ & $0.055(2)$ & 1 & 2 \\
\hline C9ME & $0.9547(12)$ & $1 / 4$ & $1.2424(6)$ & $0.065(3)$ & 1 & 2 \\
\hline $\mathrm{H} 1$ & 0.6773 & 0.0265 & 1.1694 & $0.053(16)$ & 1 & 1 \\
\hline $\mathrm{H} 2 \mathrm{~N}$ & 0.3340 & 0.1300 & 1.0726 & 0.079 & 1 & 1 \\
\hline$H 2 X$ & 0.5246 & 0.1300 & 1.0185 & 0.079 & 1 & 1 \\
\hline $\mathrm{H} 7$ & 0.3953 & 0.1223 & 1.4534 & 0.083 & 1 & 1 \\
\hline H8 & 0.4987 & -0.0073 & 1.3322 & 0.070 & 1 & 1 \\
\hline H9M1 & 1.0429 & $1 / 4$ & 1.1947 & 0.078 & 1 & 2 \\
\hline H9M2 & 0.9812 & 0.1631 & 1.2818 & 0.078 & $1 / 2$ & 1 \\
\hline H9M3 & 0.9812 & 0.3369 & 1.2818 & 0.078 & $1 / 2$ & 1 \\
\hline
\end{tabular}


.(1) 2004 American Chemical Society, J. Am. Chem. Soc., Laube ja040115t Supporting Info Page 15 Thomas Laube J. Am. Chem. Soc.

Table 2b. Anisotropic displacement parameters $\left(\AA^{2}\right)$ for $\mathrm{Me}-1^{+} \mathrm{Sb}_{2} \mathrm{~F}_{11}-$.

\begin{tabular}{|c|c|c|c|c|c|c|}
\hline Atom & $U_{11}$ & $U_{22}$ & $U_{33}$ & $U_{23}$ & $U_{13}$ & $U_{12}$ \\
\hline $\mathrm{Sb} 1$ & $0.0564(4)$ & $0.0662(5)$ & $0.0507(4)$ & $\theta$ & $0.0067(3)$ & 0 \\
\hline $\mathrm{Sb} 2$ & $0.0579(4)$ & $0.0515(4)$ & $0.0634(4)$ & $\theta$ & $0.0207(3)$ & 0 \\
\hline F1A & $0.11(2)$ & $0.20(4)$ & $0.044(15)$ & $\theta$ & $0.025(12)$ & 0 \\
\hline F11A & $0.11(2)$ & $0.20(4)$ & $0.044(15)$ & 0 & $0.025(12)$ & 0 \\
\hline$F 12 A$ & $0.17(4)$ & $0.18(5)$ & $0.14(2)$ & $-0.02(3)$ & $0.06(2)$ & $-0.12(3)$ \\
\hline F13A & $0.26(5)$ & $0.14(3)$ & $0.14(3)$ & $0.09(2)$ & $0.06(3)$ & $0.14(3)$ \\
\hline F1B & $0.116(10)$ & $0.092(14)$ & $0.039(6)$ & $0.003(5)$ & $0.008(6)$ & $0.039(9)$ \\
\hline F11B & $0.075(7)$ & $0.056(16)$ & $0.061(7)$ & $0.006(7)$ & $0.012(5)$ & $0.000(8)$ \\
\hline F12B & $0.055(6)$ & $0.10(2)$ & $0.065(6)$ & $0.005(7)$ & $-0.001(4)$ & $0.026(7)$ \\
\hline F13B & $0.133(17)$ & $0.052(8)$ & $0.094(13)$ & $-0.005(8)$ & $0.047(12)$ & $-0.016(11)$ \\
\hline F14B & $0.058(7)$ & $0.24(4)$ & $0.097(9)$ & $0.089(16)$ & $0.038(6)$ & $0.065(12)$ \\
\hline F15B & $0.30(4)$ & $0.100(16)$ & $0.104(18)$ & $0.019(13)$ & $0.012(18)$ & $-0.10(2)$ \\
\hline F21A & $0.055(15)$ & $0.15(5)$ & $0.040(14)$ & 0 & $0.009(11)$ & $\theta$ \\
\hline$F 22 A$ & $0.060(16)$ & $0.07(2)$ & $0.11(2)$ & 0 & $0.022(14)$ & 0 \\
\hline F23A & $0.11(2)$ & $0.035(9)$ & $0.15(3)$ & $-0.012(15)$ & $0.01(2)$. & $0.009(11)$ \\
\hline F24A & $0.054(15)$ & $0.06(2)$ & $0.081(19)$ & 0 & $0.006(12)$ & 0 \\
\hline $\mathrm{F} 21 \mathrm{~B}$ & $0.208(19)$ & $0.094(18)$ & $0.093(11)$ & $-0.006(9)$ & $0.102(11)$ & $0.024(17)$ \\
\hline F22B & $0.18(2)$ & $0.074(14)$ & $0.114(15)$ & $0.013(10)$ & $0.009(15)$ & $0.062(16)$ \\
\hline F23B & $0.090(12)$ & $0.19(4)$ & $0.076(11)$ & $0.005(14)$ & $-0.003(9)$ & $-0.080(14)$ \\
\hline F24B & $0.24(3)$ & $0.097(19)$ & $0.102(14)$ & $0.032(12)$ & $0.07(2)$ & $0.08(2)$ \\
\hline F25B & $0.125(18)$ & $0.22(4)$ & $0.23(2)$ & $-0.08(2)$ & $0.064(17)$ & $-0.117(19)$ \\
\hline $\mathrm{C} 1$ & $0.067(4)$ & $0.048(4)$ & $0.066(4)$ & $-0.001(3)$ & $0.017(3)$ & $0.007(3)$ \\
\hline C2 & $0.059(4)$ & $0.071(4)$ & $0.068(4)$ & $-0.002(4)$ & $0.011(3)$ & $-0.011(3)$ \\
\hline C7 & $0.054(4)$ & $0.092(5)$ & $0.058(4)$ & $0.014(4)$ & $0.001(3)$ & $-0.004(4)$ \\
\hline $\mathrm{C} 8$ & $0.049(4)$ & $0.054(4)$ & $0.069(4)$ & $0.010(3)$ & $0.001(3)$ & $-0.005(3)$ \\
\hline C8A & $0.051(3)$ & $0.053(4)$ & $0.066(4)$ & $0.000(3)$ & $0.013(3)$ & $0.003(3)$ \\
\hline $\mathrm{Cg}$ & $0.056(5)$ & $0.042(5)$ & $0.062(5)$ & $\theta$ & $0.000(4)$ & 0 \\
\hline C9ME & $0.039(5)$ & $0.080(7)$ & $0.073(6)$ & 0 & $0.001(4)$ & 0 \\
\hline
\end{tabular}

Table 2c. Principal mean square atomic displacements $U$

\begin{tabular}{ccccc}
\hline Atom & $U_{\max }$ & $U_{\operatorname{med}}$ & $U_{\min }$ & $U_{\max } / U_{\min }$ \\
\hline Sb1 & 0.0662 & 0.0577 & 0.0502 & 1.32 \\
Sb2 & 0.0700 & 0.0515 & 0.0477 & 1.47 \\
F1A & 0.2034 & 0.1069 & 0.0386 & 5.27
\end{tabular}


(1) 2004 American Chemical Society, J. Am. Chem. Soc., Laube ja040115t Supporting Info Page 16 Thomas Laube J.Am. Chem. Soc.

\begin{tabular}{ccccc} 
F11A & 0.2034 & 0.1069 & 0.0386 & 5.27 \\
F12A & 0.3037 & 0.1302 & 0.0500 & 6.07 \\
F13A & 0.3702 & 0.1413 & 0.0259 & 14.29 \\
F1B & 0.1456 & 0.0638 & 0.0389 & 3.74 \\
F11B & 0.0751 & 0.0649 & 0.0517 & 1.45 \\
F12B & 0.1122 & 0.0713 & 0.0404 & 2.78 \\
F13B & 0.1460 & 0.0753 & 0.0490 & 2.98 \\
F14B & 0.2915 & 0.0539 & 0.0362 & 8.05 \\
F15B & 0.3517 & 0.1076 & 0.0486 & 7.24 \\
F21A & 0.1476 & 0.0546 & 0.0397 & 3.72 \\
F22A & 0.1148 & 0.0654 & 0.0578 & 1.99 \\
F23A & 0.1509 & 0.1139 & 0.0320 & 4.72 \\
F24A & 0.0830 & 0.0597 & 0.0535 & 1.55 \\
F21B & 0.2376 & 0.0975 & 0.0340 & 6.99 \\
F22B & 0.2136 & 0.1179 & 0.0440 & 4.85 \\
F23B & 0.2389 & 0.0798 & 0.0448 & 5.33 \\
F24B & 0.2820 & 0.0848 & 0.0615 & 4.59 \\
F25B & 0.3453 & 0.1749 & 0.0461 & 7.49 \\
C1 & 0.0723 & 0.0610 & 0.0447 & 1.62 \\
C2 & 0.0777 & 0.0680 & 0.0528 & 1.47 \\
C7 & 0.0991 & 0.0601 & 0.0477 & 2.08 \\
C8 & 0.0800 & 0.0492 & 0.0460 & 1.74 \\
C8A & 0.0664 & 0.0547 & 0.0478 & 1.39 \\
C9 & 0.0712 & 0.0507 & 0.0422 & 1.69 \\
C9ME & 0.0798 & 0.0763 & 0.0384 & 2.08 \\
\hline & & & & \\
\hline
\end{tabular}

Table 3. Bond lengths $(\AA)$ and angles (deg) for $\mathrm{Me}^{-1} \mathrm{Sb}_{2} \mathrm{~F}_{11}{ }^{-}$.

\begin{tabular}{ll|ll}
\hline & & & \\
$\mathrm{Sb}(1)-\mathrm{F}(15 \mathrm{~B})$ & $1.783(13)$ & $\mathrm{Sb}(2)-\mathrm{F}(25 \mathrm{~B})$ & $1.822(12)$ \\
$\mathrm{Sb}(1)-\mathrm{F}(15 \mathrm{~B}) \# 1$ & $1.783(13)$ & $\mathrm{Sb}(2)-\mathrm{F}(25 \mathrm{~B}) \# 1$ & $1.822(12)$ \\
$\mathrm{Sb}(1)-\mathrm{F}(12 \mathrm{~B}) \# 1$ & $1.817(9)$ & $\mathrm{Sb}(2)-\mathrm{F}(23 \mathrm{~A})$ & $1.835(11)$ \\
$\mathrm{Sb}(1)-\mathrm{F}(12 \mathrm{~B})$ & $1.817(9)$ & $\mathrm{Sb}(2)-\mathrm{F}(23 \mathrm{~A}) \# 1$ & $1.835(11)$ \\
$\mathrm{Sb}(1)-\mathrm{F}(11 \mathrm{~B}) \# 1$ & $1.847(9)$ & $\mathrm{Sb}(2)-\mathrm{F}(23 \mathrm{~B}) \# 1$ & $1.829(10)$ \\
$\mathrm{Sb}(1)-\mathrm{F}(11 \mathrm{~B})$ & $1.847(9)$ & $\mathrm{Sb}(2)-\mathrm{F}(23 \mathrm{~B})$ & $1.829(11)$ \\
$\mathrm{Sb}(1)-\mathrm{F}(13 \mathrm{~A}) \# 1$ & $1.832(14)$ & $\mathrm{Sb}(2)-\mathrm{F}(21 \mathrm{~B}) \# 1$ & $1.863(10)$ \\
$\mathrm{Sb}(1)-\mathrm{F}(13 \mathrm{~A})$ & $1.832(13)$ & $\mathrm{Sb}(2)-\mathrm{F}(21 \mathrm{~B})$ & $1.863(10)$ \\
$\mathrm{Sb}(1)-\mathrm{F}(12 \mathrm{~A})$ & $1.837(13)$ & $\mathrm{Sb}(2)-\mathrm{F}(22 \mathrm{~B})$ & $1.857(12)$ \\
$\mathrm{Sb}(1)-\mathrm{F}(12 \mathrm{~A}) \# 1$ & $1.837(13)$ & $\mathrm{F}(11 \mathrm{~A})-\mathrm{C}(9)$ & $3.034(19)$ \\
$\mathrm{Sb}(1)-\mathrm{F}(14 \mathrm{~B})$ & $1.872(10)$ & $\mathrm{F}(11 \mathrm{~B})-\mathrm{C}(9)$ & $3.161(13)$ \\
$\mathrm{Sb}(1)-\mathrm{F}(14 \mathrm{~B}) \# 1$ & $1.872(10)$ & $\mathrm{C}(1)-\mathrm{C}(9)$ & $1.465(8)$ \\
$\mathrm{Sb}(2)-\mathrm{F}(22 \mathrm{~A})$ & $1.818(15)$ & $\mathrm{C}(1)-\mathrm{C}(8 \mathrm{~A})$ & $1.496(8)$ \\
$\mathrm{Sb}(2)-\mathrm{F}(24 \mathrm{~B})$ & $1.817(12)$ & $\mathrm{C}(1)-\mathrm{C}(2)$ & $1.556(9)$ \\
$\mathrm{Sb}(2)-\mathrm{F}(24 \mathrm{~B}) \# 1$ & $1.817(13)$ & $\mathrm{C}(2)-\mathrm{C}(2) \# 1$ & $1.507(13)$ \\
& & &
\end{tabular}


-(C) 2004 American Chemical Society, J. Am. Chem. Soc., Laube ja040115t Supporting Info Page 17 Thomas Laube J. Am. Chem. Soc.

$\begin{array}{ll}C(7)-C(8) & 1.358(9) \\ C(7)-C(7) \# 1 & 1.423(15) \\ C(7)-F(21 A) \# 2 & 3.65(3) \\ C(7)-F(21 B) \# 2 & 4.03(2) \\ C(8)-C(8 A) & 1.395(8) \\ C(8)-F(21 A) \# 2 & 3.84(2) \\ C(8)-F(21 B) \# 2 & 4.12(2) \\ C(8 A)-C(8 A) \# 1 & 1.414(12)\end{array}$

$F(15 B)-S b(1)-F(15 B) \# 1$ $F(15 B)-S b(1)-F(12 B) \# 1$ $F(15 B) \# 1-S b(1)-F(12 B) \# 1$ $F(15 B)-S b(1)-F(12 B)$ $F(15 B) \# 1-S b(1)-F(12 B)$ $F(12 B) \# 1-S b(1)-F(12 B)$ $F(15 B)-S b(1)-F(11 B) \# 1$ $F(15 B) \# 1-S b(1)-F(11 B) \# 1$ $F(12 B) \# 1-S b(1)-F(11 B) \# 1$ $F(12 B)-S b(1)-F(11 B) \# 1$ $F(15 B)-S b(1)-F(11 B)$ $F(15 B) \# 1-S b(1)-F(11 B)$

$F(12 B) \# 1-S b(1)-F(11 B)$ $F(12 B)-S b(1)-F(11 B)$ $F(11 B) \# 1-S b(1)-F(11 B)$ $F(15 B)-S b(1)-F(13 A) \# 1$ $F(15 B) \# 1-S b(1)-F(13 A) \# 1$ $F(12 B) \# 1-S b(1)-F(13 A) \# 1$ $F(12 B)-S b(1)-F(13 A) \# 1$ $F(11 B) \# 1-S b(1)-F(13 A) \# 1$ $F(11 B)-S b(1)-F(13 A) \# 1$ $F(15 B)-S b(1)-F(13 A)$ $F(15 B) \# 1-S b(1)-F(13 A)$ $F(12 B) \# 1-S b(1)-F(13 A)$ $F(12 B)-S b(1)-F(13 A)$ $F(11 B) \# 1-S b(1)-F(13 A)$ $F(11 B)-S b(1)-F(13 A)$ $F(13 A) \# 1-S b(1)-F(13 A)$ $F(15 B)-S b(1)-F(12 A)$ $F(15 B) \# 1-S b(1)-F(12 A)$ $F(12 B) \# 1-S b(1)-F(12 A)$ $F(12 B)-S b(1)-F(12 A)$ $F(11 B) \# 1-S b(1)-F(12 A)$ $F(11 B)-S b(1)-F(12 A)$ $F(13 A) \# 1-S b(1)-F(12 A)$ $F(13 A)-S b(1)-F(12 A)$ $F(15 B)-S b(1)-F(12 A) \# 1$ $F(15 B) \# 1-S b(1)-F(12 A) \# 1$ $F(12 B) \# 1-S b(1)-F(12 A) \# 1$ $F(12 B)-S b(1)-F(12 A) \# 1$ $F(11 B) \# 1-S b(1)-F(12 A) \# 1$ $F(11 B)-S b(1)-F(12 A) \# 1$ $F(13 A) \# 1-S b(1)-F(12 A) \# 1$ $F(13 A)-S b(1)-F(12 A) \# 1$ $F(12 A)-S b(1)-F(12 A) \# 1$ $F(15 B)-S b(1)-F(14 B)$ $F(15 B) \# 1-S b(1)-F(14 B)$ $F(12 B) \# 1-S b(1)-F(14 B)$ $F(12 B)-S b(1)-F(14 B)$ $F(11 B) \# 1-S b(1)-F(14 B)$ $F(11 B)-S b(1)-F(14 B)$ $F(13 A) \# 1-S b(1)-F(14 B)$ $F(13 A)-S b(1)-F(14 B)$
$141.1(14)$

114.1(12)

$91.9(7)$

$91.9(7)$

$114.1(12)$

$23.0(10)$

$108.2(12)$

$97.3(8)$

$94.5(5)$

$96.8(6)$

$97.3(8)$

$108.2(12)$

$96.8(6)$

94.5(5)

$11.3(17)$

$29.5(11)$

$117.0(10)$

$143.2(7)$

$121.4(8)$

$103.1(11)$

$95.1(13)$

$117.0(10)$

$29.5(11)$

$121.4(8)$

$143.2(7)$

$95.1(13)$

$103.1(11)$

89.2(11)

$149.7(10)$

$61.4(10)$

$35.6(7)$

$58.0(7)$

$81.2(10)$

$89.3(11)$

$175.7(9)$

$89.9(5)$

$61.4(10)$

$149.7(10)$

$58.0(7)$

$35.6(7)$

89.3(11)

$81.2(10)$

$89.9(5)$

$175.7(9)$

$90.7(10)$

89.9(7)

$59.9(13)$

$151.7(9)$

$170.4(5)$

$91.6(6)$

94.6(5)

$60.6(10)$

$30.3(8)$
$C(8 A)-C(9)$

$C(8 A)-F(21 A) \# 2$

$1.897(10)$

$C(8 A)-F(21 B) \# 2$

$C(9)-C(1) \# 1$

$C(9)-C(9 M E)$

$C(9)-C(8 A) \# 1$

$C(9)-F(21 A) \# 2$

$C(9)-F(21 B) \# 2$

$4.09(2)$

$4.41(2)$

$1.465(8)$

$1.479(11)$

$1.897(10)$

$4.119(19)$

$4.386(18)$
$F(12 A)-S b(1)-F(14 B)$
$F(12 A) \# 1-S b(1)-F(14 B)$
$F(15 B)-S b(1)-F(14 B) \# 1$
$F(15 B) \# 1-S b(1)-F(14 B) \# 1$
$F(12 B) \# 1-S b(1)-F(14 B) \# 1$
$F(12 B)-S b(1)-F(14 B) \# 1$
$F(11 B) \# 1-S b(1)-F(14 B) \# 1$
$F(11 B)-S b(1)-F(14 B) \# 1$
$F(13 A) \# 1-S b(1)-F(14 B) \# 1$
$F(13 A)-S b(1)-F(14 B) \# 1$
$F(12 A)-S b(1)-F(14 B) \# 1$
$F(12 A) \# 1-S b(1)-F(14 B) \# 1$
$F(14 B)-S b(1)-F(14 B) \# 1$
$F(22 A)-S b(2)-F(24 B)$
$F(22 A)-S b(2)-F(24 B) \# 1$
$F(24 B)-S b(2)-F(24 B) \# 1$
$F(22 A)-S b(2)-F(25 B)$
$F(24 B)-S b(2)-F(25 B)$
$F(24 B) \# 1-S b(2)-F(25 B)$
$F(22 A)-S b(2)-F(25 B) \# 1$
$F(24 B)-S b(2)-F(25 B) \# 1$
$F(24 B) \# 1-S b(2)-F(25 B) \# 1$
$F(25 B)-S b(2)-F(25 B) \# 1$
$F(22 A)-S b(2)-F(23 A)$
$F(24 B)-S b(2)-F(23 A)$
$F(24 B) \# 1-\mathrm{Sb}(2)-F(23 A)$
$F(25 B)-S b(2)-F(23 A)$
$F(25 B) \# 1-S b(2)-F(23 A)$
$F(22 A)-S b(2)-F(23 A) \# 1$
$F(24 B)-S b(2)-F(23 A) \# 1$
$F(24 B) \# 1-S b(2)-F(23 A) \# 1$
$F(25 B)-S b(2)-F(23 A) \# 1$
$F(25 B) \# 1-S b(2)-F(23 A) \# 1$
$F(23 A)-S b(2)-F(23 A) \# 1$
$F(22 A)-S b(2)-F(23 B) \# 1$
$F(24 B)-S b(2)-F(23 B) \# 1$
$F(24 B) \# 1-S b(2)-F(23 B) \# 1$
$F(25 B)-S b(2)-F(23 B) \# 1$
$F(25 B) \# 1-S b(2)-F(23 B) \# 1$
$F(23 A)-S b(2)-F(23 B) \# 1$
$F(23 A) \# 1-S b(2)-F(23 B) \# 1$
$F(22 A)-S b(2)-F(23 B)$
$F(24 B)-S b(2)-F(23 B)$
$F(24 B) \# 1-S b(2)-F(23 B)$
$F(25 B)-S b(2)-F(23 B)$
$F(25 B) \# 1-\mathrm{Sb}(2)-F(23 B)$
$\mathrm{F}(23 \mathrm{~A})-\mathrm{Sb}(2)-\mathrm{F}(23 \mathrm{~B})$
$F(23 A) \# 1-S b(2)-F(23 B)$
$F(23 B) \# 1-S b(2)-F(23 B)$
$F(22 A)-S b(2)-F(21 B) \# 1$
$F(24 B)-S b(2)-F(21 B) \# 1$
$F(24 B) \# 1-S b(2)-F(21 B) \# 1$
$F(25 B)-S b(2)-F(21 B) \# 1$
119.2(9)
$149.9(9)$
$59.9(13)$
$89.9(7)$
$170.4(5)$
$151.7(9)$
$94.6(5)$
$91.6(6)$
$30.3(8)$
$60.6(10)$
$149.9(9)$
$119.2(9)$
$30.7(14)$
$114.8(9)$
$114.8(9)$
$126.6(18)$
$26.3(9)$
$91.3(7)$
$140.7(17)$
$26.3(9)$
$140.7(17)$
$91.3(7)$
$49.7(18)$
$89.7(5)$
28.5(10)
$154.7(11)$
$64.3(10)$
$113.9(10)$
$89.7(5)$
$154.7(11)$
$28.5(10)$
$113.9(10)$
$64.3(10)$
$172.6(12)$
154.4(9)
$40.7(15)$
$90.7(8)$
$128.5(15)$
$170.1(7)$
$64.7(10)$
$115.7(10)$
$154.4(9)$
$90.7(8)$
$40.7(15)$
$170.1(7)$
$128.5(15)$
$115.7(10)$
$64.7(10)$
$51.1(17)$
$81.6(11)$
$74.0(10)$
$94.8(7)$
$85.5(8)$ 
- (C) 2004 American Chemical Society, J. Am. Chem. Soc., Laube ja040115t Supporting Info Page 18 Thomas Laube J. Am. Chem. Soc.

\begin{tabular}{|c|c|c|c|}
\hline 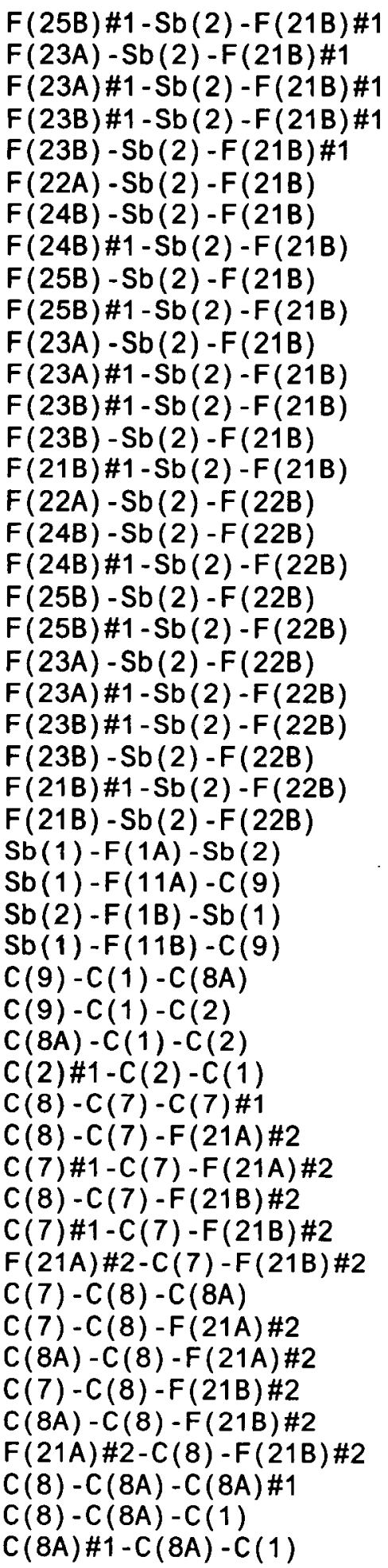 & $\begin{array}{c}95.2(7) \\
82.0(9) \\
105.2(9) \\
94.3(6) \\
104.3(8) \\
81.6(11) \\
94.8(7) \\
74.0(10) \\
95.2(7) \\
85.5(8) \\
105.2(9) \\
82.0(9) \\
104.3(8) \\
94.3(7) \\
23.2(14) \\
67.2(9) \\
170.4(6) \\
56.2(15) \\
88.2(7) \\
40.9(14) \\
147.0(10) \\
27.9(8) \\
135.4(14) \\
88.2(6) \\
115.5(10) \\
94.8(7) \\
168.2(17) \\
171(2) \\
154.3(7) \\
156.1(8) \\
79.7(5) \\
106.4(6) \\
111.8(5) \\
105.0(3) \\
121.5(4) \\
87.6(4) \\
78.75(16) \\
84.4(4) \\
85.2(4) \\
6.5(3) \\
117.5(6) \\
71.7(5) \\
90.1(4) \\
76.5(4) \\
92.6(4) \\
7.2(3) \\
120.8(4) \\
130.8(6) \\
107.5(3)\end{array}$ & $\begin{array}{l}C(8)-C(8 A)-C(9) \\
C(8 A) \# 1-C(8 A)-C(9) \\
C(1)-C(8 A)-C(9) \\
C(8)-C(8 A)-F(21 A) \# 2 \\
C(8 A) \# 1-C(8 A)-F(21 A) \# 2 \\
C(1)-C(8 A)-F(21 A) \# 2 \\
C(9)-C(8 A)-F(21 A) \# 2 \\
C(8)-C(8 A)-F(21 B) \# 2 \\
C(8 A) \# 1-C(8 A)-F(21 B) \# 2 \\
C(1)-C(8 A)-F(21 B) \# 2 \\
C(9)-C(8 A)-F(21 B) \# 2 \\
F(21 A) \# 2-C(8 A)-F(21 B) \# 2 \\
C(1)-C(9)-C(1) \# 1 \\
C(1)-C(9)-C(9 M E) \\
C(1) \# 1-C(9)-C(9 M E) \\
C(1)-C(9)-C(8 A) \\
C(1) \# 1-C(9)-C(8 A) \\
C(9 M E)-C(9)-C(8 A) \\
C(1)-C(9)-C(8 A) \# 1 \\
C(1) \# 1-C(9)-C(8 A) \# 1 \\
C(9 M E)-C(9)-C(8 A) \# 1 \\
C(8 A)-C(9)-C(8 A) \# 1 \\
C(1)-C(9)-F(11 A) \\
C(1) \# 1-C(9)-F(11 A) \\
C(9 M E)-C(9)-F(11 A) \\
C(8 A)-C(9)-F(11 A) \\
C(8 A) \# 1-C(9)-F(11 A) \\
C(1)-C(9)-F(11 B) \\
C(1) \# 1-C(9)-F(11 B) \\
C(9 M E)-C(9)-F(11 B) \\
C(8 A)-C(9)-F(11 B) \\
C(8 A) \# 1-C(9)-F(11 B) \\
F(11 A)-C(9)-F(11 B) \\
C(1)-C(9)-F(21 A) \# 2 \\
C(1) \# 1-C(9)-F(21 A) \# 2 \\
C(9 M E)-C(9)-F(21 A) \# 2 \\
C(8 A)-C(9)-F(21 A) \# 2 \\
C(8 A) \# 1-C(9)-F(21 A) \# 2 \\
F(11 A)-C(9)-F(21 A) \# 2 \\
F(11 B)-C(9)-F(21 A) \# 2 \\
C(1)-C(9)-F(21 B) \# 2 \\
C(1) \# 1-C(9)-F(21 B) \# 2 \\
C(9 M E)-C(9)-F(21 B) \# 2 \\
C(8 A)-C(9)-F(21 B) \# 2 \\
C(8 A) \# 1-C(9)-F(21 B) \# 2 \\
F(11 A)-C(9)-F(21 B) \# 2 \\
F(11 B)-C(9)-F(21 B) \# 2 \\
F(21 A) \# 2-C(9)-F(21 B) \# 2 \\
C\left(\begin{array}{l} \\
C\end{array}\right)\end{array}$ & $\begin{array}{r}143.1(5) \\
68.1(2) \\
49.5(4) \\
69.9(4) \\
80.04(11) \\
113.0(5) \\
77.6(5) \\
69.0(4) \\
85.7(3) \\
108.5(4) \\
76.8(4) \\
6.4(3) \\
104.2(8) \\
127.9(4) \\
127.9(4) \\
50.9(4) \\
87.5(5) \\
123.0(6) \\
87.5(5) \\
50.9(4) \\
123.0(6) \\
43.7(4) \\
90.1(7) \\
90.1(7) \\
90.1(10) \\
138.5(8) \\
138.5(8) \\
90.4(6) \\
95.6(6) \\
85.5(6) \\
140.3(6) \\
144.3(5) \\
5.7(8) \\
112.4(5) \\
112.4(5) \\
51.5(6) \\
75.7(5) \\
75.7(5) \\
141.6(10) \\
136.8(5) \\
110.5(5) \\
119.1(5) \\
46.8(5) \\
78.3(4) \\
82.0(4) \\
136.5(9) \\
131.4(5) \\
6.9(4)\end{array}$ \\
\hline
\end{tabular}

Symmetry transformations used to generate equivalent atoms:

\#1 $x,-y+1 / 2, z$ (mirror plane to obtain the second half of the cation and of the anion)

\#2 $x, y, z+1$ (translation to obtain the second anion in "syn" position with regard to $\mathrm{C9}$ ) 
(c) 2004 American Chemical Society, J. Am. Chem. Soc., Laube ja040115t Supporting Info Page 19 Thomas Laube J. Am. Chem. Soc.

Table 4. Torsion angles (deg) for $\mathrm{Me}^{-1} \mathrm{Sb}_{2} \mathrm{~F}_{11}{ }^{-}$.

$\begin{array}{ll}\text { F15B-Sb1-F1A-Sb2 } & 72.9(9) \\ \text { F15B\#1-Sb1-F1A-Sb2 } & -72.9(9) \\ \text { F12B\#1-Sb1-F1A-Sb2 } & -167.9(5) \\ \text { F12B-Sb1-F1A-Sb2 } & 167.9(5) \\ \text { F11B\#1-Sb1-F1A-Sb2 } & -155(4) \\ \text { F11B-Sb1-F1A-Sb2 } & 155(4) \\ \text { F13A\#1-Sb1-F1A-Sb2 } & 44.6(5) \\ \text { F13A-Sb1-F1A-Sb2 } & -44.6(5) \\ \text { F12A-Sb1-F1A-Sb2 } & -134.5(5) \\ \text { F12A\#1-Sb1-F1A-Sb2 } & 134.5(5) \\ \text { F14B-Sb1-F1A-Sb2 } & -15.6(7) \\ \text { F14B\#1-Sb1-F1A-Sb2 } & 15.6(7) \\ \text { F22A-Sb2-F1A-Sb1 } & 180.00(2) \\ \text { F24B-Sb2-F1A-Sb1 } & 65.3(10) \\ \text { F24B\#1-Sb2-F1A-Sb1 } & -65.3(10) \\ \text { F25B-Sb2-F1A-Sb1 } & 154.7(9) \\ \text { F25B\#1-Sb2-F1A-Sb1 } & -154.7(9) \\ \text { F23A-Sb2-F1A-Sb1 } & 90.1(5) \\ \text { F23A\#1-Sb2-F1A-Sb1 } & -90.1(5) \\ \text { F23B\#1-Sb2-F1A-Sb1 } & 25.6(8) \\ \text { F23B-Sb2-F1A-Sb1 } & -25.6(8) \\ \text { F21B\#1-Sb2-F1A-Sb1 } & 134(3) \\ \text { F21B-Sb2-F1A-Sb1 } & -134(3) \\ \text { F22B-Sb2-F1A-Sb1 } & -113.3(10) \\ \text { F15B-Sb1-F11A-C9 } & -73.5(9) \\ \text { F15B\#1-Sb1-F11A-C9 } & 73.5(9) \\ \text { F12B\#1-Sb1-F11A-C9 } & 168.2(5) \\ \text { F12B-Sb1-F11A-C9 } & -168.2(5) \\ \text { F11B\#1-Sb1-F11A-C9 } & 146(7) \\ \text { F11B-Sb1-F11A-C9 } & -146(7) \\ \text { F13A\#1-Sb1-F11A-C9 } & -44.7(6) \\ \text { F13A-Sb1-F11A-C9 } & 44.7(6) \\ \text { F12A-Sb1-F11A-C9 } & 134.6(5) \\ \text { F12A\#1-Sb1-F11A-C9 } & -134.6(5) \\ \text { F14B-Sb1-F11A-C9 } & 15.4(7) \\ \text { F14B\#1-Sb1-F11A-C9 } & -15.4(7) \\ \text { F22A-Sb2-F1B-Sb1 } & -62.3(19) \\ \text { F24B-Sb2-F1B-Sb1 } & -176.7(18) \\ \text { F24B\#1-Sb2-F1B-Sb1 } & 57(2) \\ \text { F25B-Sb2-F1B-Sb1 } & -85.0(18) \\ \text { F25B\#1-Sb2-F1B-Sb1 } & -36(2) \\ \text { F23A-Sb2-F1B-Sb1 } & -150(2) \\ \text { F23A\#1-Sb2-F1B-Sb1 } & 28.6(19) \\ \text { F23B\#1-Sb2-F1B-Sb1 } & 143(2) \\ \text { F23B-Sb2-F1B-Sb1 } & 92.2(18) \\ \text { F21B\#1-Sb2-F1B-Sb1 } & -152(2) \\ \text { F21B-Sb2-F1B-Sb1 } & 88(58) \\ \text { F22B-Sb2-F1B-Sb1 } & 3.6(18) \\ \text { F15B-Sb1-F1B-Sb2 } & -43.9(19) \\ \text { F15B\#1-Sb1-F1B-Sb2 } & 166(2) \\ \text { F12B\#1-Sb1-F1B-Sb2 } & 71(2) \\ \text { F12B-Sb1-F1B-Sb2 } & 48.1(18) \\ \text { F11B\#1-Sb1-F1B-Sb2 } & 150.0(19) \\ \text { F11B-Sb1-F1B-Sb2 } & 149(4) \\ \text { F13A\#1-Sb1-F1B-Sb2 } & -73.5(19) \\ & \end{array}$

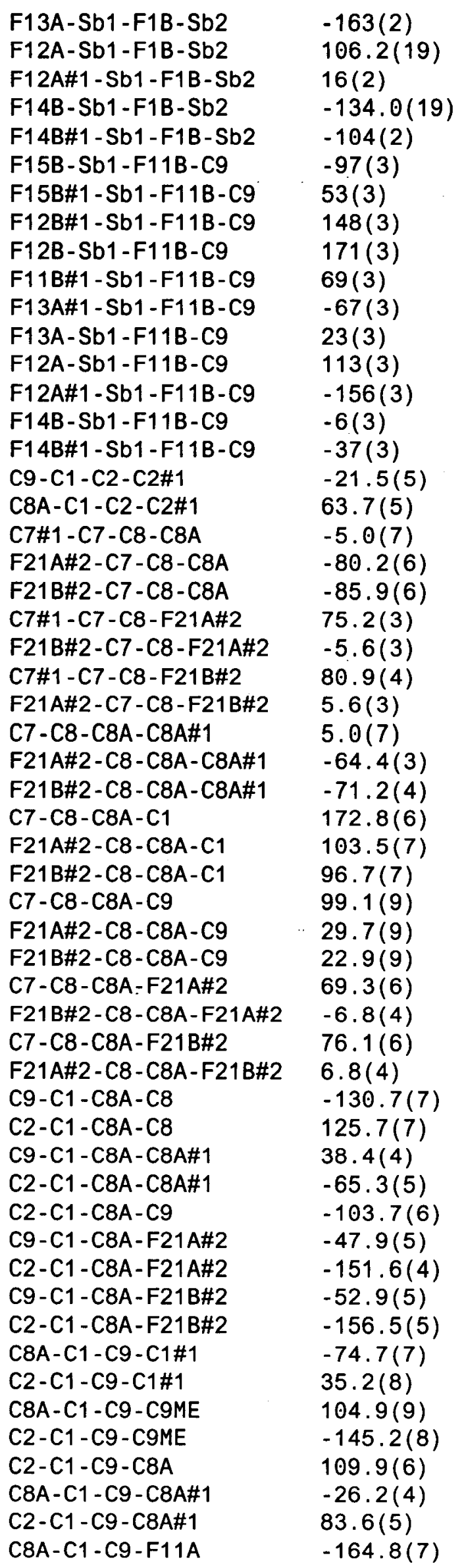


. (C) 2004 American Chemical Society, J. Am. Chem. Soc., Laube ja040115t Supporting Info Page 20 Thomas Laube J. Am. Chem. Soc.

$\begin{array}{ll}\text { C2-C1-C9-F11A } & -55.0(8) \\ \text { C8A-C1-C9-F11B } & -170.6(5) \\ \text { C2-C1-C9-F11B } & -60.7(6) \\ \text { C8A-C1-C9-F21A\#2 } & 47.2(6) \\ \text { C2-C1-C9-F21A\#2 } & 157.0(5) \\ \text { C8A-C1-C9-F21B\#2 } & 54.3(5) \\ \text { C2-C1-C9-F21B\#2 } & 164.2(4) \\ \text { C8-C8A-C9-C1 } & 107.1(10) \\ \text { C8A\#1-C8A-C9-C1 } & -140.3(5) \\ \text { F21A\#2-C8A-C9-C1 } & 135.6(5) \\ \text { F21B\#2-C8A-C9-C1 } & 129.1(5) \\ \text { C8-C8A-C9-C1\#1 } & -142.3(9) \\ \text { C8A\#1-C8A-C9-C1\#1 } & -29.7(3) \\ \text { C1-C8A-C9-C1\#1 } & 110.6(8) \\ \text { F21A\#2-C8A-C9-C1\#1 } & -113.8(4) \\ \text { F21B\#2-C8A-C9-C1\#1 } & -120.4(5) \\ \text { C8-C8A-C9-C9ME } & -7.4(10) \\ \text { C8A\#1-C8A-C9-C9ME } & 105.1(4) \\ \text { C1-C8A-C9-C9ME } & -114.5(5) \\ \text { F21A\#2-C8A-C9-C9ME } & 21.0(4) \\ \text { F21B\#2-C8A-C9-C9ME } & 14.5(5) \\ \text { C8-C8A-C9-C8A\#1 } & -112.6(8) \\ \text { C1-C8A-C9-C8A\#1 } & 140.3(5) \\ \text { F21A\#2-C8A-C9-C8A\#1 } & -84.1(2) \\ \text { F21B\#2-C8A-C9-C8A\#1 } & -90.6(3) \\ \text { C8-C8A-C9-F11A } & 130.4(11) \\ \text { C8A\#1-C8A-C9-F11A } & -117.0(8) \\ \text { C1-C8A-C9-F11A } & 23.3(8) \\ \text { F21A\#2-C8A-C9-F11A } & 158.9(8) \\ \text { F21B\#2-C8A-C9-F11A } & 152.3(8) \\ & \end{array}$

$\begin{array}{ll}\text { C8-C8A-C9-F11B } & 122.0(11) \\ \text { C8A\#1-C8A-C9-F11B } & -125.4(8) \\ \text { C1-C8A-C9-F11B } & 14.9(8) \\ \text { F21A\#2-C8A-C9-F11B } & 150.4(8) \\ \text { F21B\#2-C8A-C9-F11B } & 143.9(8) \\ \text { C8-C8A-C9-F21A\#2 } & -28.5(8) \\ \text { C8A\#1-C8A-C9-F21A\#2 } & 84.1(2) \\ \text { C1-C8A-C9-F21A\#2 } & -135.6(5) \\ \text { F21B\#2-C8A-C9-F21A\#2 } & -6.5(3) \\ \text { C8-C8A-C9-F21B\#2 } & -22.0(9) \\ \text { C8A\#1-C8A-C9-F21B\#2 } & 90.6(3) \\ \text { C1-C8A-C9-F21B\#2 } & -129.1(5) \\ \text { F21A\#2-C8A-C9-F21B\#2 } & 6.5(3) \\ \text { Sb1-F11A-C9-C1 } & 52.1(4) \\ \text { Sb1-F11A-C9-C1\#1 } & -52.1(4) \\ \text { Sb1-F11A-C9-C9ME } & 180.00(6) \\ \text { Sb1-F11A-C9-C8A } & 34.2(7) \\ \text { Sb1-F11A-C9-C8A\#1 } & -34.2(7) \\ \text { Sb1-F11A-C9-F11B } & 145(8) \\ \text { Sb1-F11A-C9-F21A\#2 } & 180.00(6) \\ \text { Sb1-F11A-C9-F21B\#2 } & 172.9(5) \\ \text { Sb1-F11B-C9-C1 } & 74(3) \\ \text { Sb1-F11B-C9-C1\#1 } & -30(3) \\ \text { Sb1-F11B-C9-C9ME } & -158(3) \\ \text { Sb1-F11B-C9-C8A } & 63(4) \\ \text { Sb1-F11B-C9-C8A\#1 } & -12(4) \\ \text { Sb1-F11B-C9-F11A } & -13(5) \\ \text { Sb1-F11B-C9-F21A\#2 } & -162(3) \\ \text { Sb1-F11B-C9-F21B\#2 } & -168(3) \\ & \end{array}$

Symmetry transformations used to generate equivalent atoms:

\#1 $x,-y+1 / 2, z$ (mirror plane to obtain the second half of the cation and of the anion)

\#2 $x, y, z+1 \quad$ (translation to obtain the second anion in "syn" position with regard to $\mathrm{C} 9$ ) 


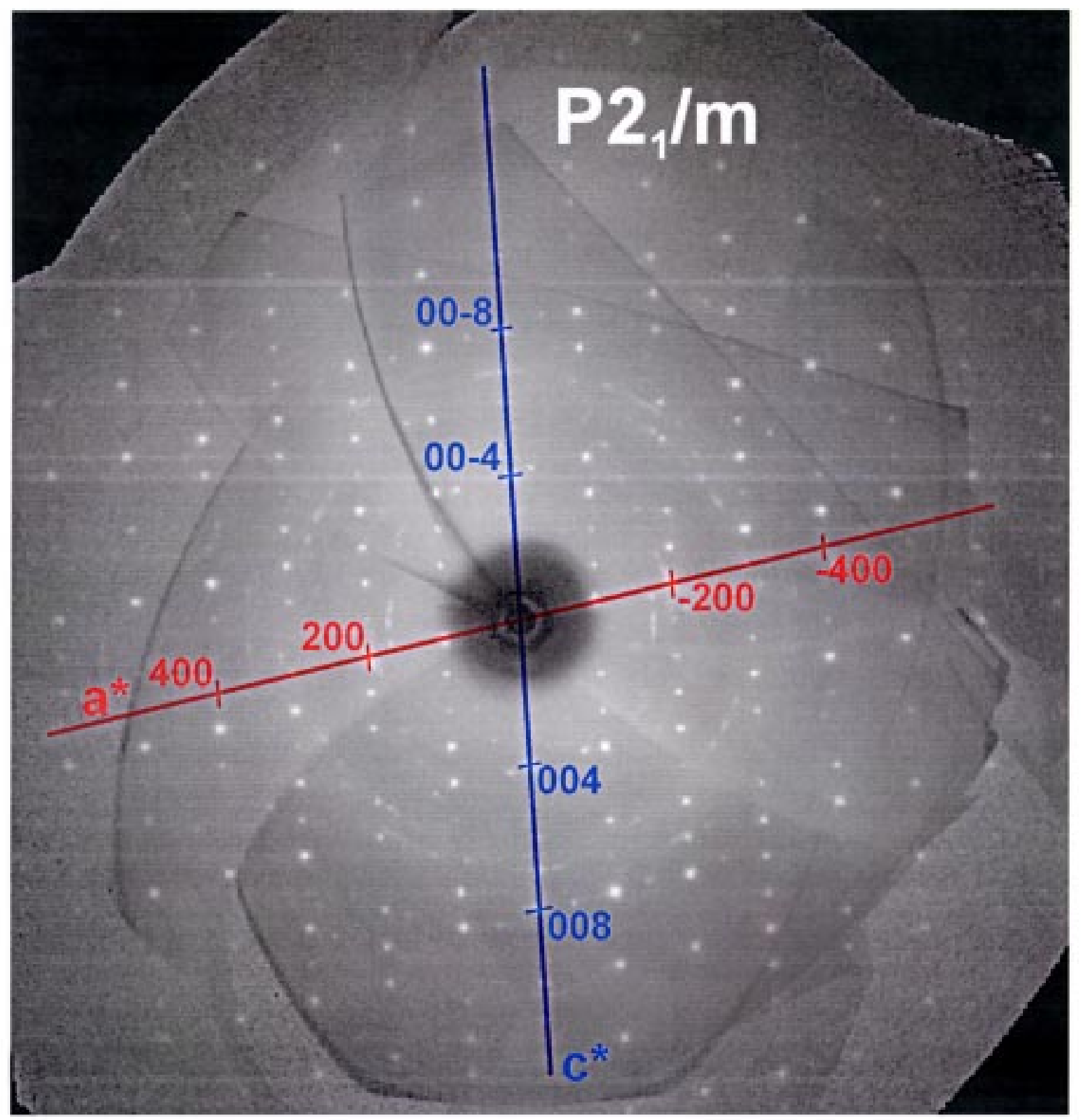

Simulated precession image of the $h 0 l$ plane showing diffraction rings from powdery components on the measured single crystal of $\mathrm{Me}^{-1} \mathrm{Sb}_{2} \mathrm{~F}_{11}{ }^{-}$. The labeling of the image was carried out with the help of additional diagrams ${ }^{62}$ showing the reciprocal space with axes and the reflections with the Miller indices. 


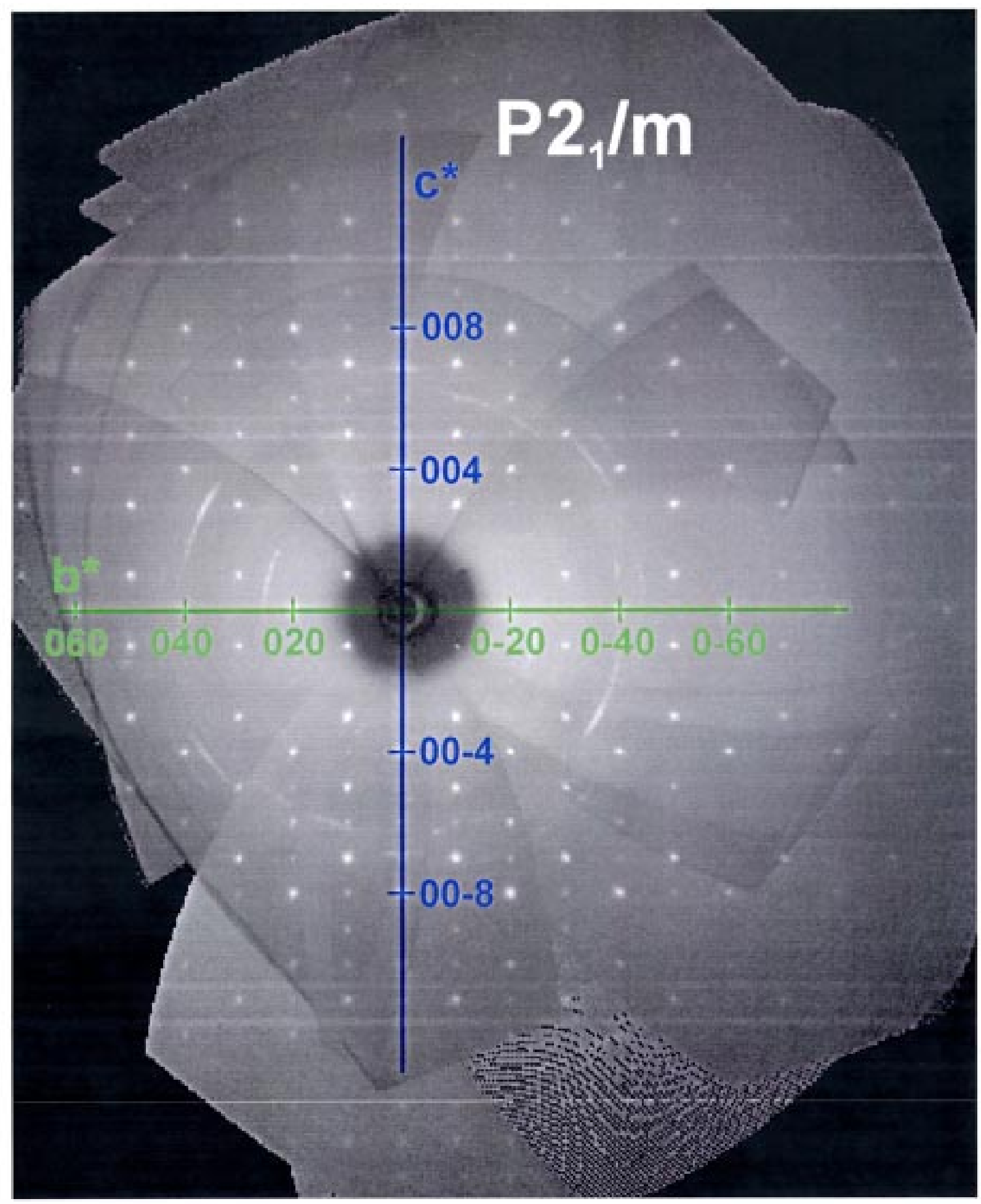

Simulated precession images of the $0 \mathrm{kl}$ plane showing diffraction rings from powdery components on the measured single crystal of $\mathrm{Me}^{-} \mathbf{1}^{+} \mathrm{Sb}_{2} \mathrm{~F}_{11}{ }^{-}$. The labeling of the image was carried out with the help of additional diagrams ${ }^{62}$ showing the reciprocal space with axes and the reflections with the Miller indices. 
Calculated $\mathrm{Mo}_{\kappa \alpha}$ powder diffraction profile in $\boldsymbol{P} \mathbf{2}_{1} / \mathbf{m}$ (PowderCell 2.3; some peaks are labelled with $h \boldsymbol{k l}$ )
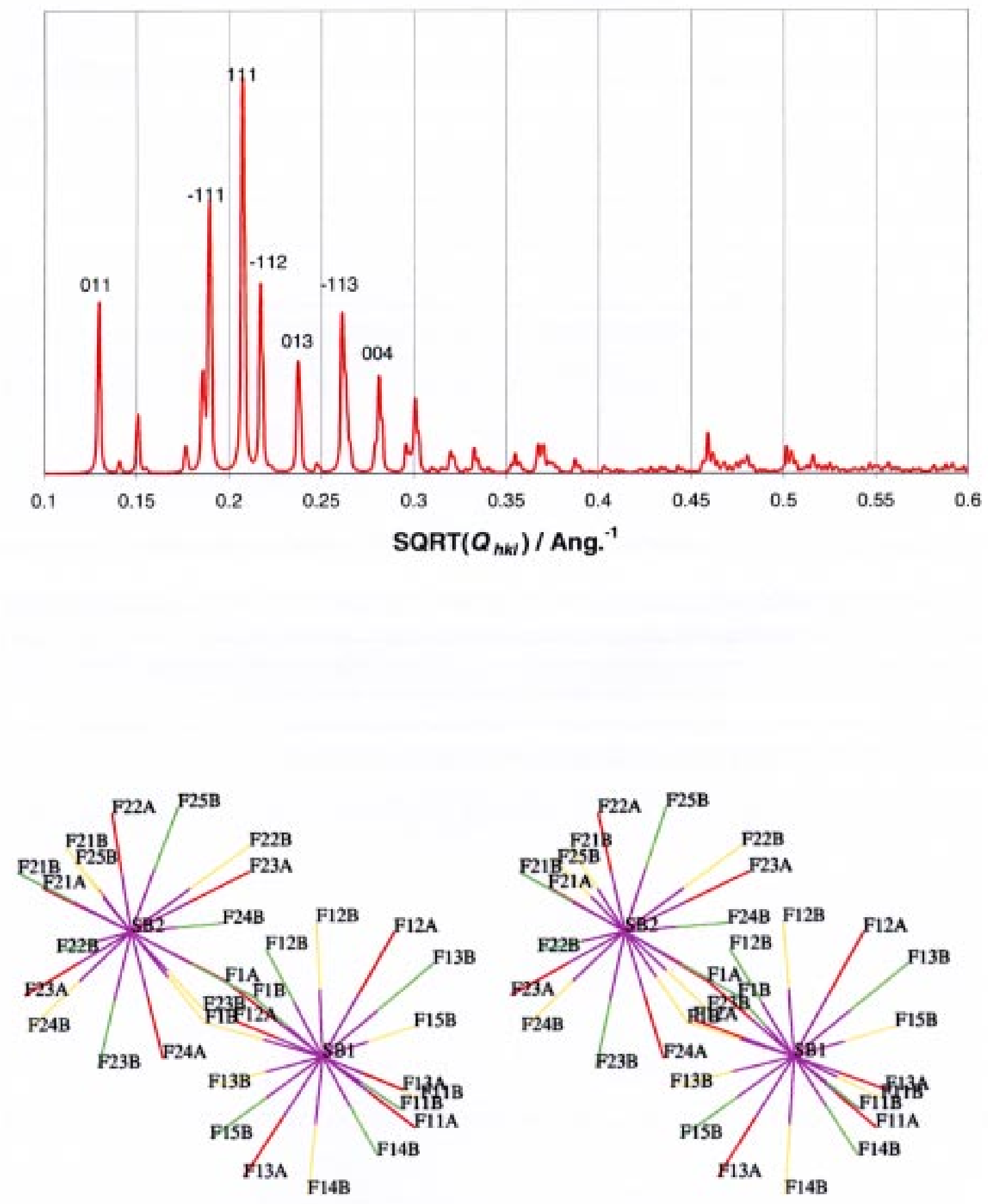

Stereo diagram ${ }^{64}$ of the three disordered orientation of the $\mathrm{Sb} 2 \mathrm{~F} 11$ anion in the structure of Me-1 ${ }^{+} \mathrm{Sb}_{2} \mathrm{~F}_{11}{ }^{-}$. The anion with red $\mathrm{F}$ atoms lies on the mirror plane (names ending with $\mathrm{A}$; sof $=0.28$ ), the anions with green and yellow $\mathrm{F}$ atom are related by the mirror plane (names ending with $B$; each $F$ site of the has sof $=0.36$ ). 

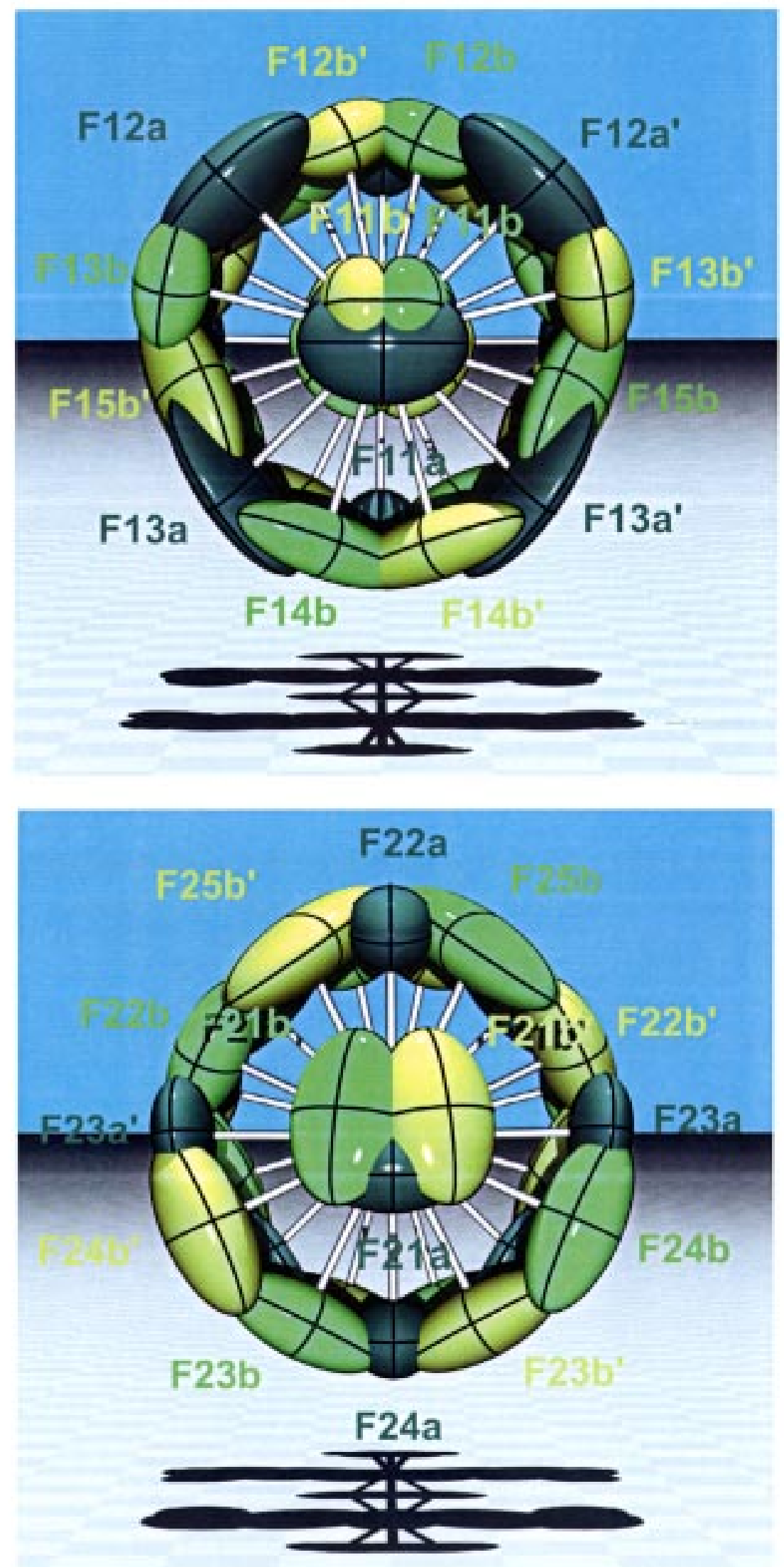

ORTEP diagrams looking approximately along F11-Sb1 (top) and F21-Sb2 (bottom) in the crystal structure of Me-1 ${ }^{+} \mathrm{Sb}_{2} \mathrm{~F}_{11}{ }^{-}$. For the colour scheme see Figure 1 of the manuscript. 


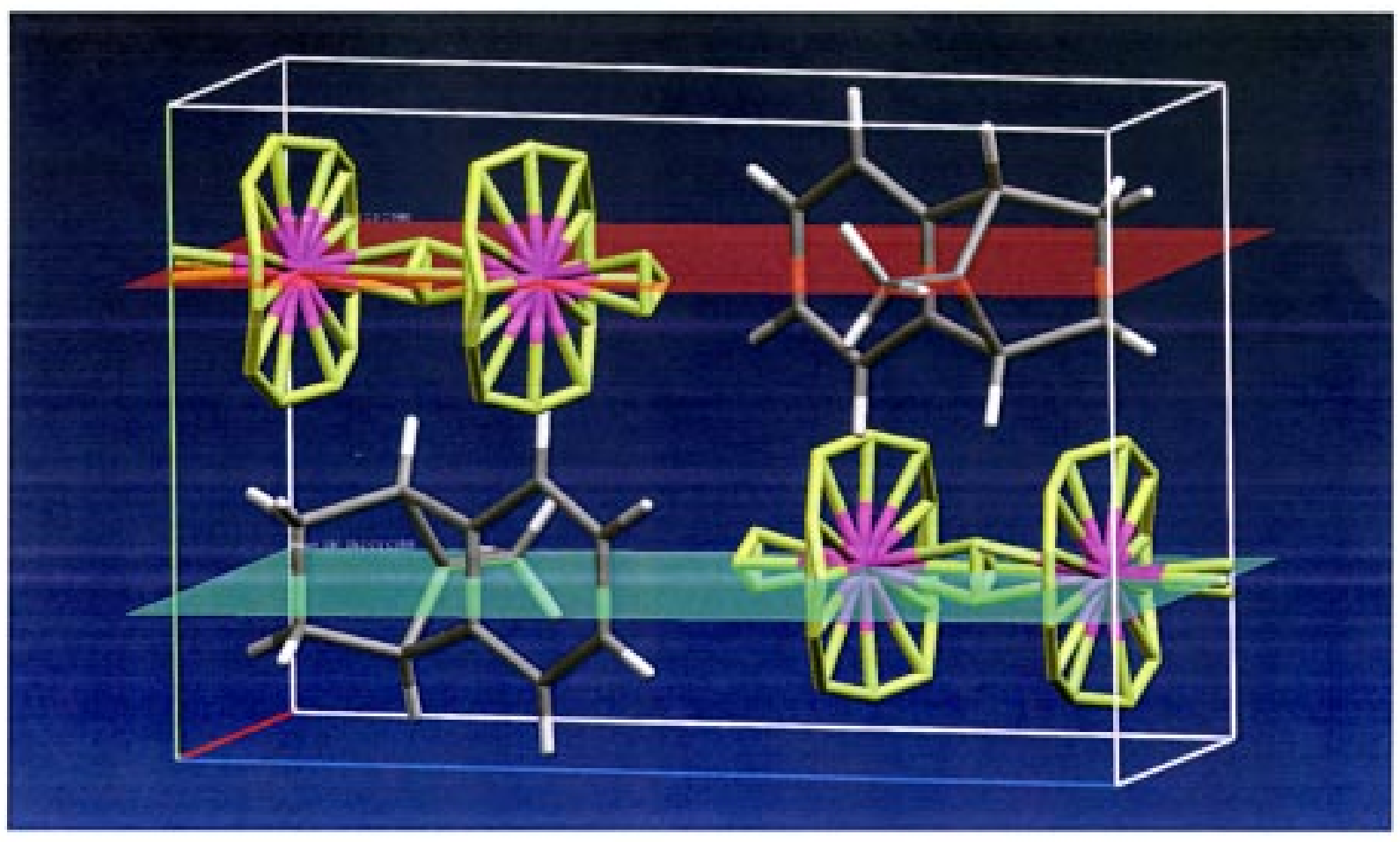

Unit cell of the crystal structure of $\mathrm{Me}-1^{+} \mathrm{Sb}_{2} \mathrm{~F}_{11}{ }^{-}$with the mirror planes $\left(P{ }_{1} / m\right.$; axes: $a$, $h$, c). Short distances between $\mathrm{F}$ atoms belonging to different anion orientations are drawn as bonds because this feature cannot be suppressed by the graphics program.

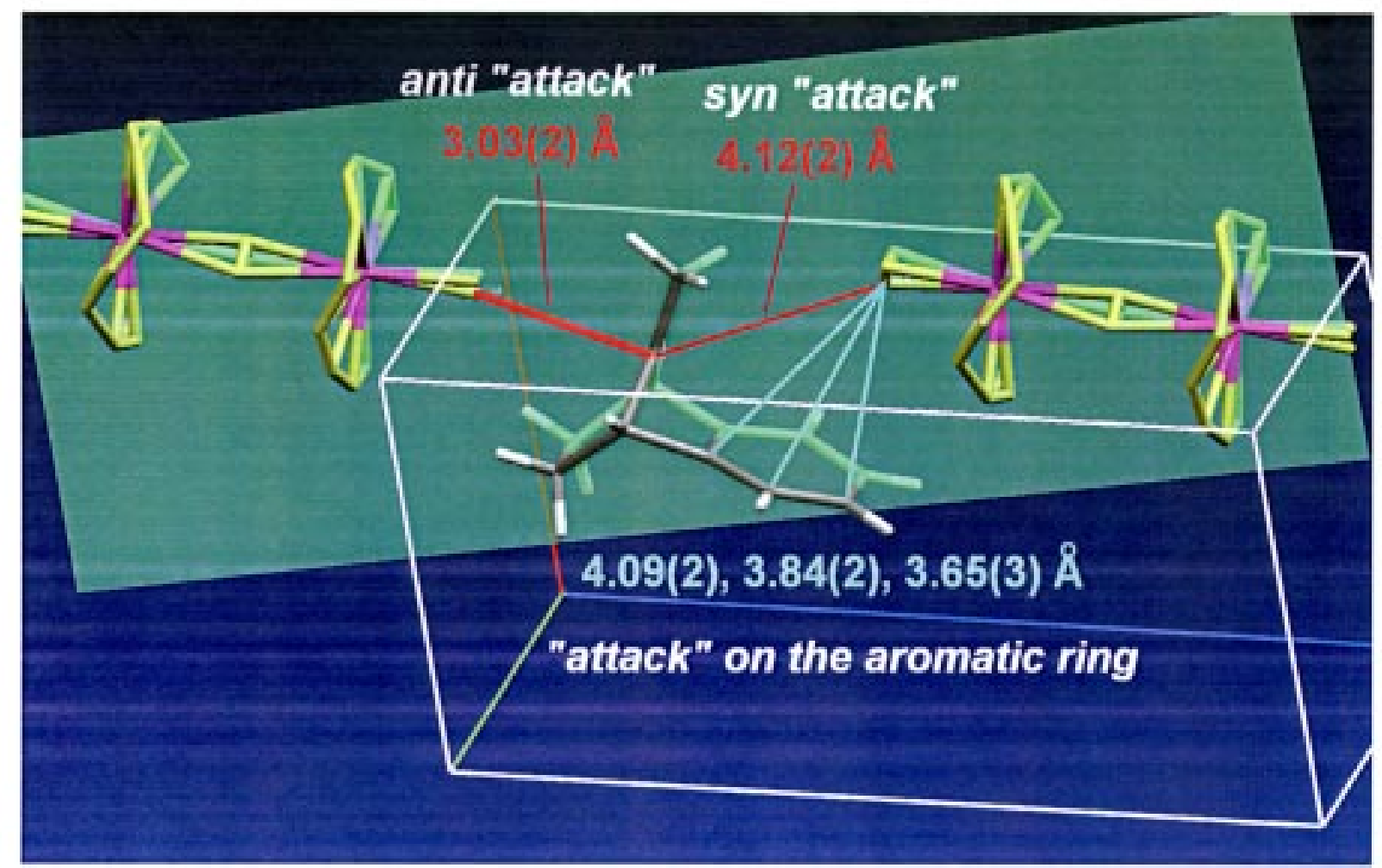

Interpretation of the closest cation-anion contacts in the crystal structure of $\mathrm{Me}-\mathrm{I}^{+} \mathrm{Sb}_{2} \mathrm{~F}_{11}{ }^{-}$ 


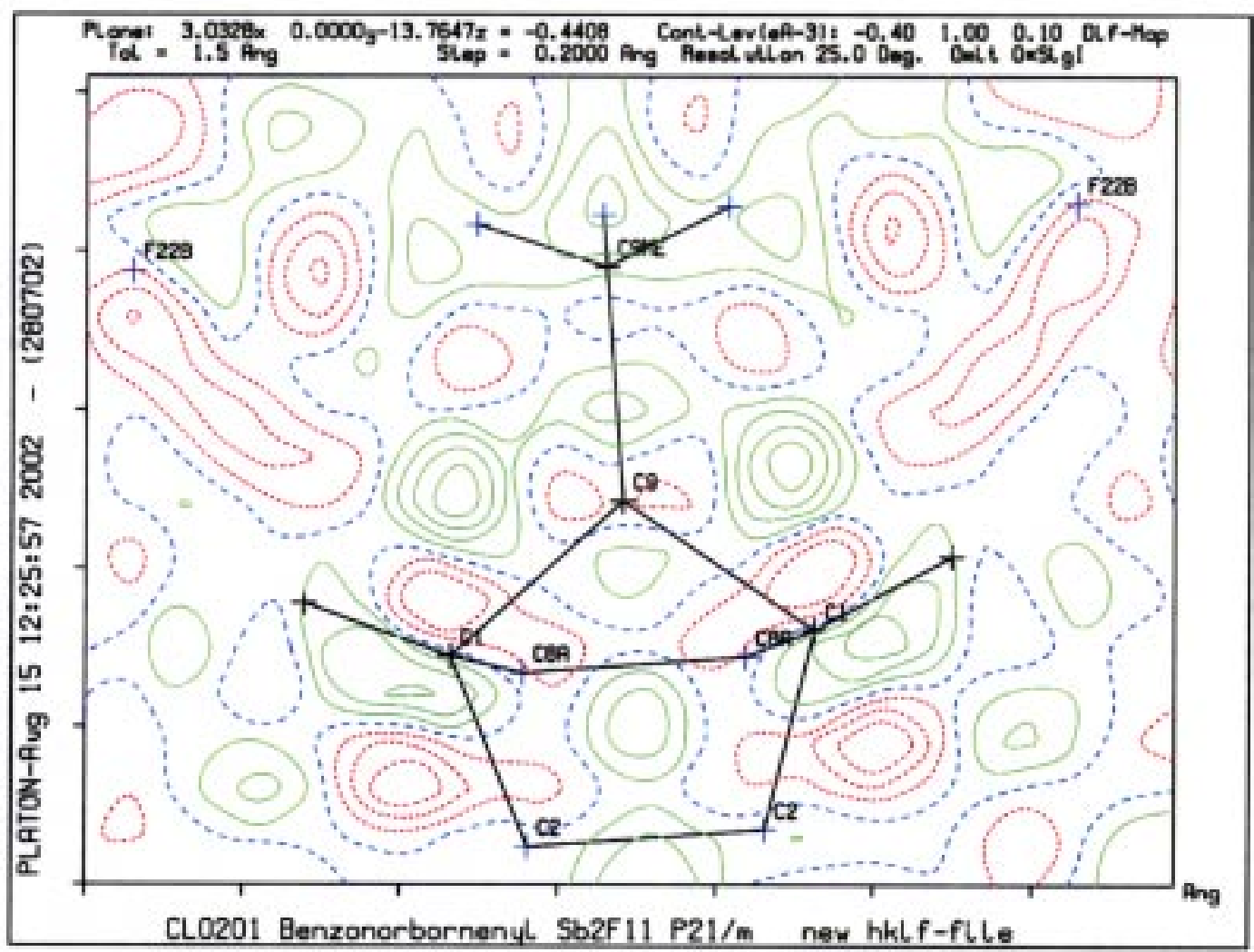

Difference electron density $\Delta \rho$ in the plane defined by $\mathrm{C} 1, \mathrm{C} 4(=\mathrm{Cl} \# 1), \mathrm{C} 9$ with $\Delta \Delta \rho=0.1 \mathrm{e}$ $\AA^{-3}$ (positive: solid green; zero: dashed blue; negative: dotted red).

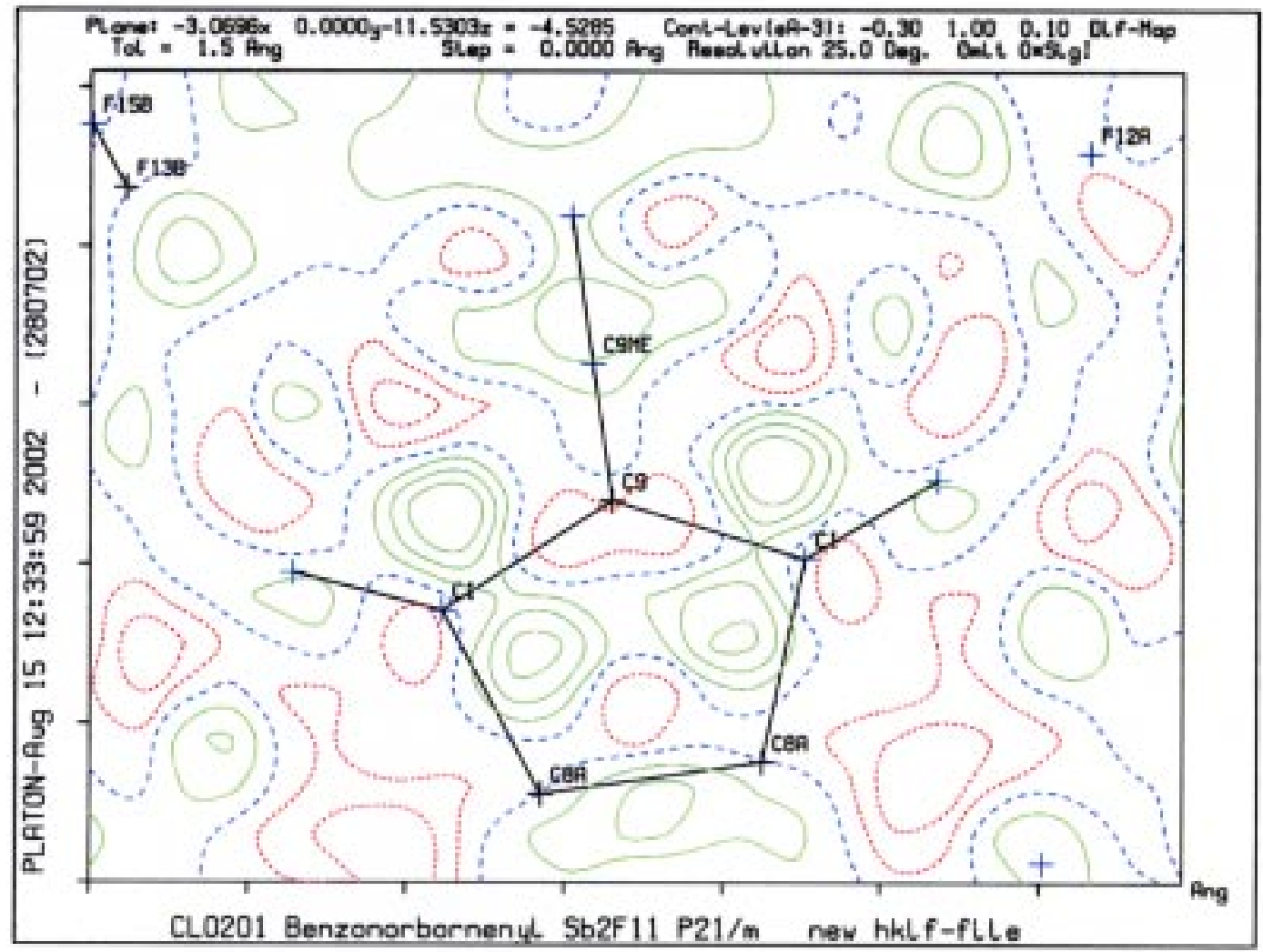

Difference electron density $\Delta \rho$ in the plane defined by $\mathrm{C} 4 \mathrm{a}(=\mathrm{C} 8 \mathrm{a} \# 1), \mathrm{C} 8 \mathrm{a}, \mathrm{C} 9$ with $\Delta \Delta \rho=$ 0.1 e $\AA^{-3}$ (positive: solid green; zero: dashed blue; negative: dotted red). 


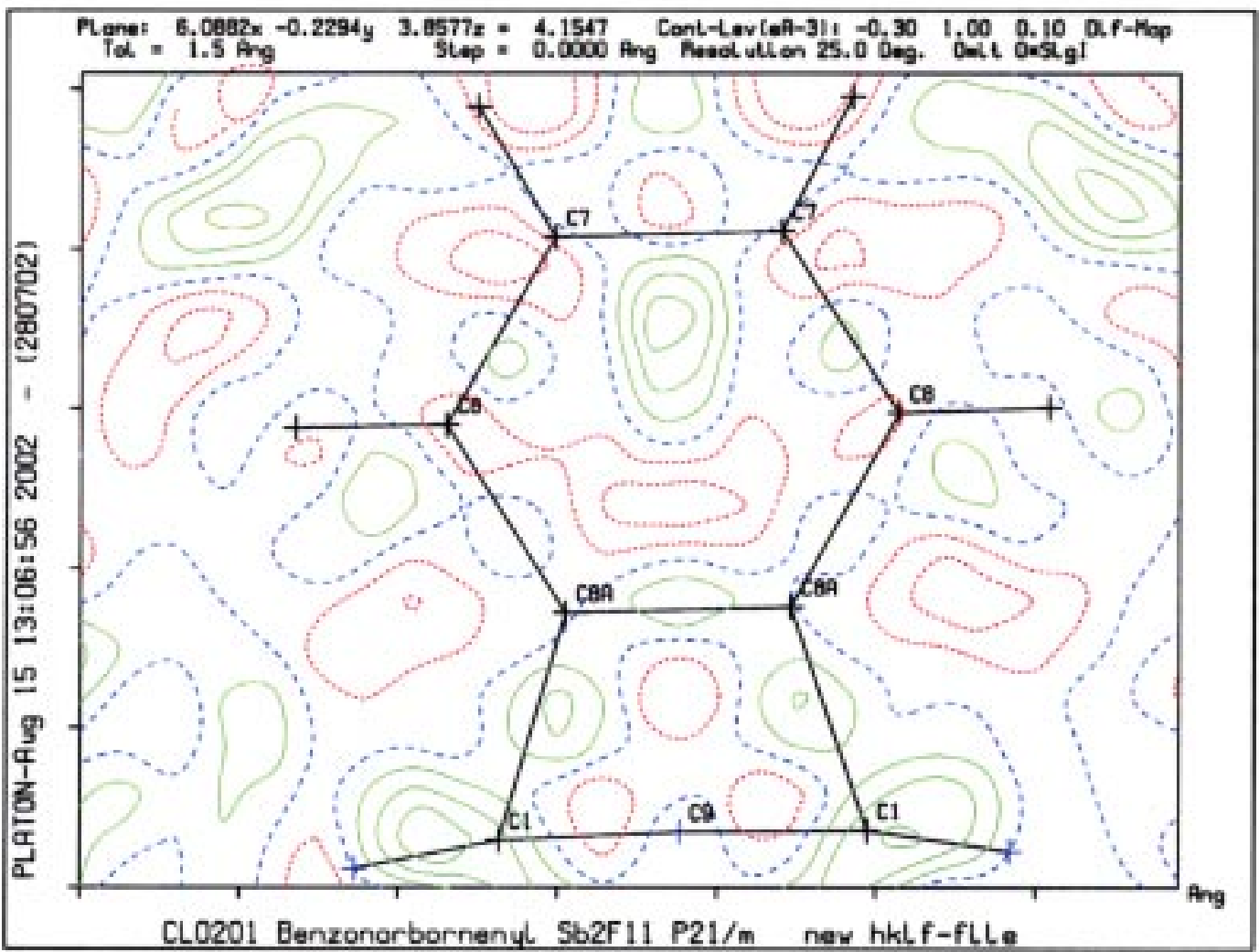

Difference electron density $\Delta \rho$ in the plane defined by $\mathrm{C} 5$ (= C8\#1), C7, C8a (aromatic plane) with $\Delta \Delta \rho=0.1 \mathrm{e} \AA^{-3}$ (positive: solid green; zero: dashed blue; negative: dotted red).

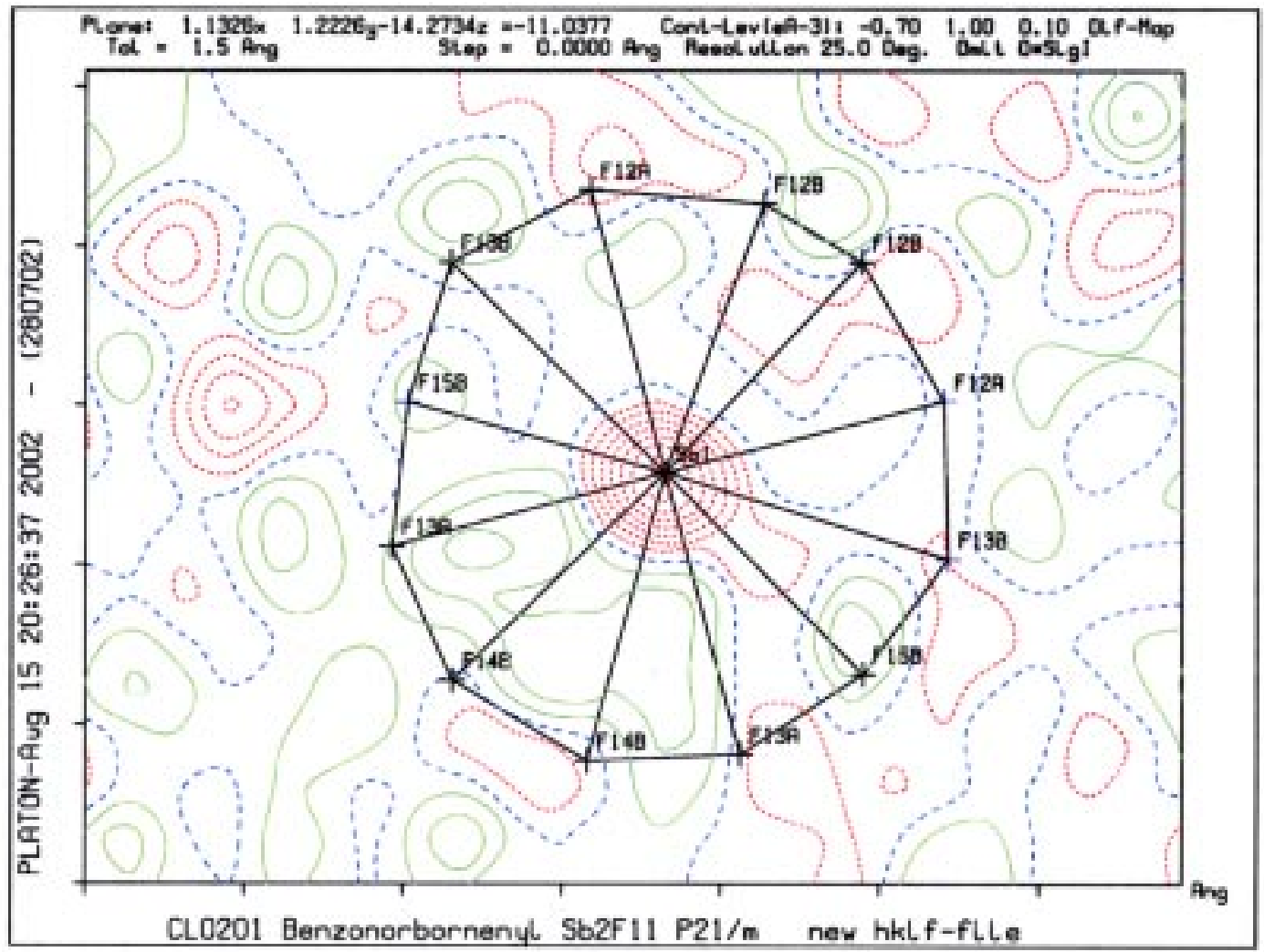

Difference electron density $\Delta \rho$ in the plane defined by F12F, F13B, F14B with $\Delta \Delta \rho=0.1$ e $\AA$ ${ }^{3}$ (positive: solid green; zero: dashed blue; negative: dotted red). 


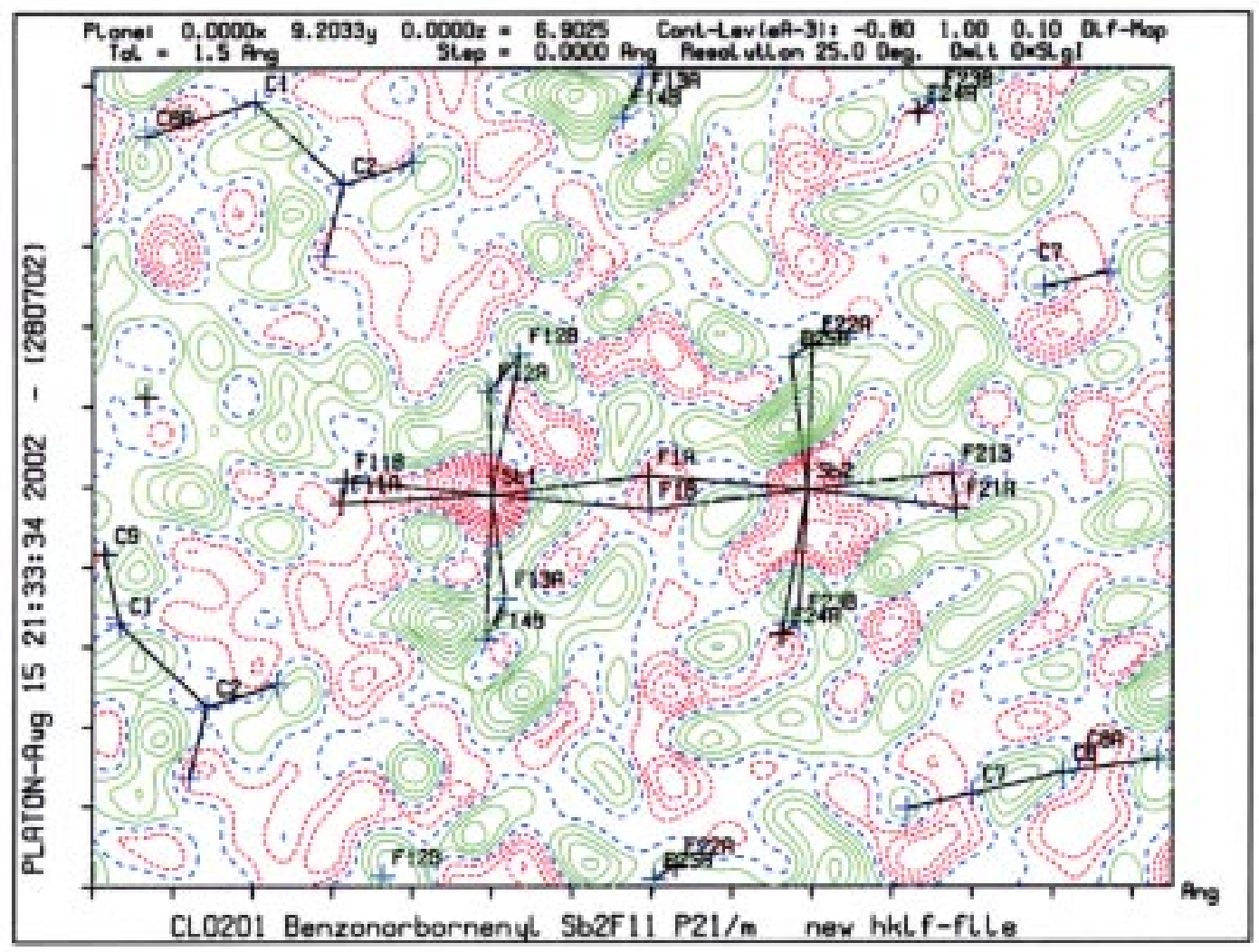

Difference electron density $\Delta \rho$ in the plane defined by F1a, F11a, F21a with $\Delta \Delta \rho=0.1$ e $\AA^{-3}$ (positive: solid green; zero: dashed blue; negative: dotted red).

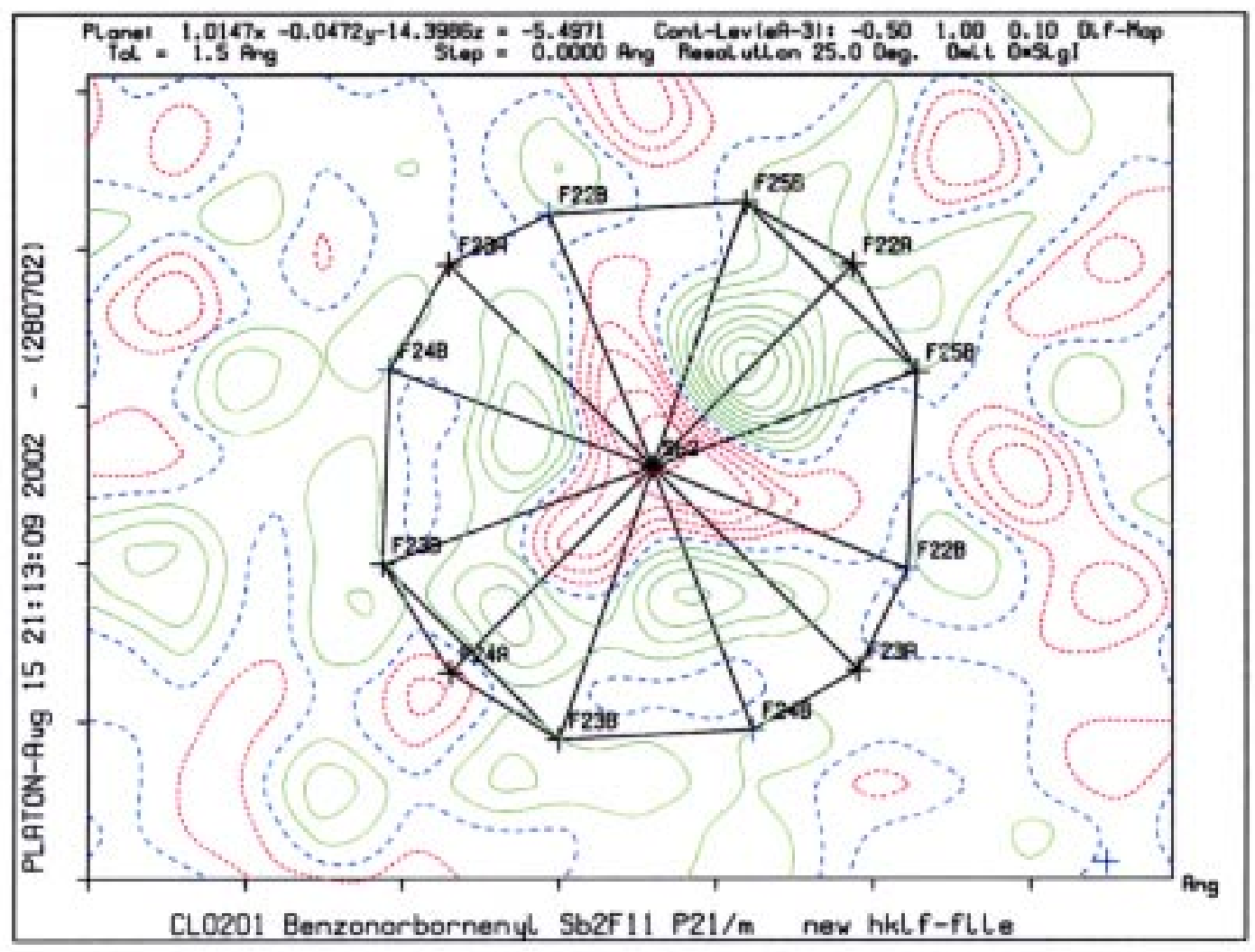

Difference electron density $\Delta \rho$ in the plane defined by F22a, F23a, F24a with $\Delta \Delta \rho=0.1$ e $\AA^{-3}$ (positive: solid green; zero: dashed blue; negative: dotted red). 


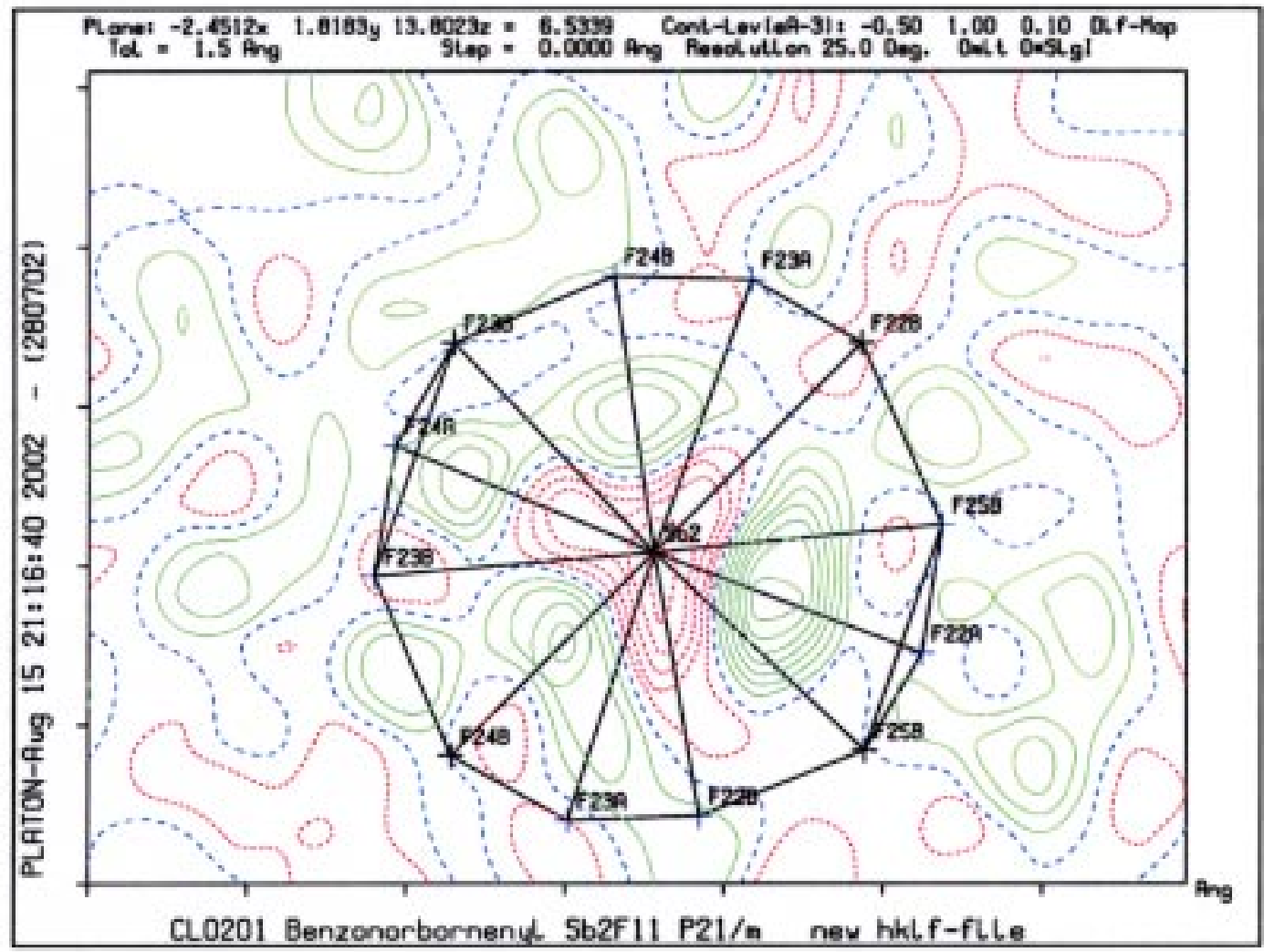

Difference electron density $\Delta \rho$ in the plane defined by F22b, F23b, F24b with $\Delta \Delta \rho=0.1$ e $\AA^{-3}$ (positive: solid green; zero: dashed blue; negative: dotted red). 
( () 2004 American Chemical Society, J. Am. Chem. Soc., Laube ja040115t Supporting Info Page 30 Thomas Laube J.Am. Chem. Soc.

\section{Some photos showing the preparation of $\mathrm{Me}-1^{+} \mathrm{Sb}_{2} \mathrm{~F}_{11}{ }^{-}$}

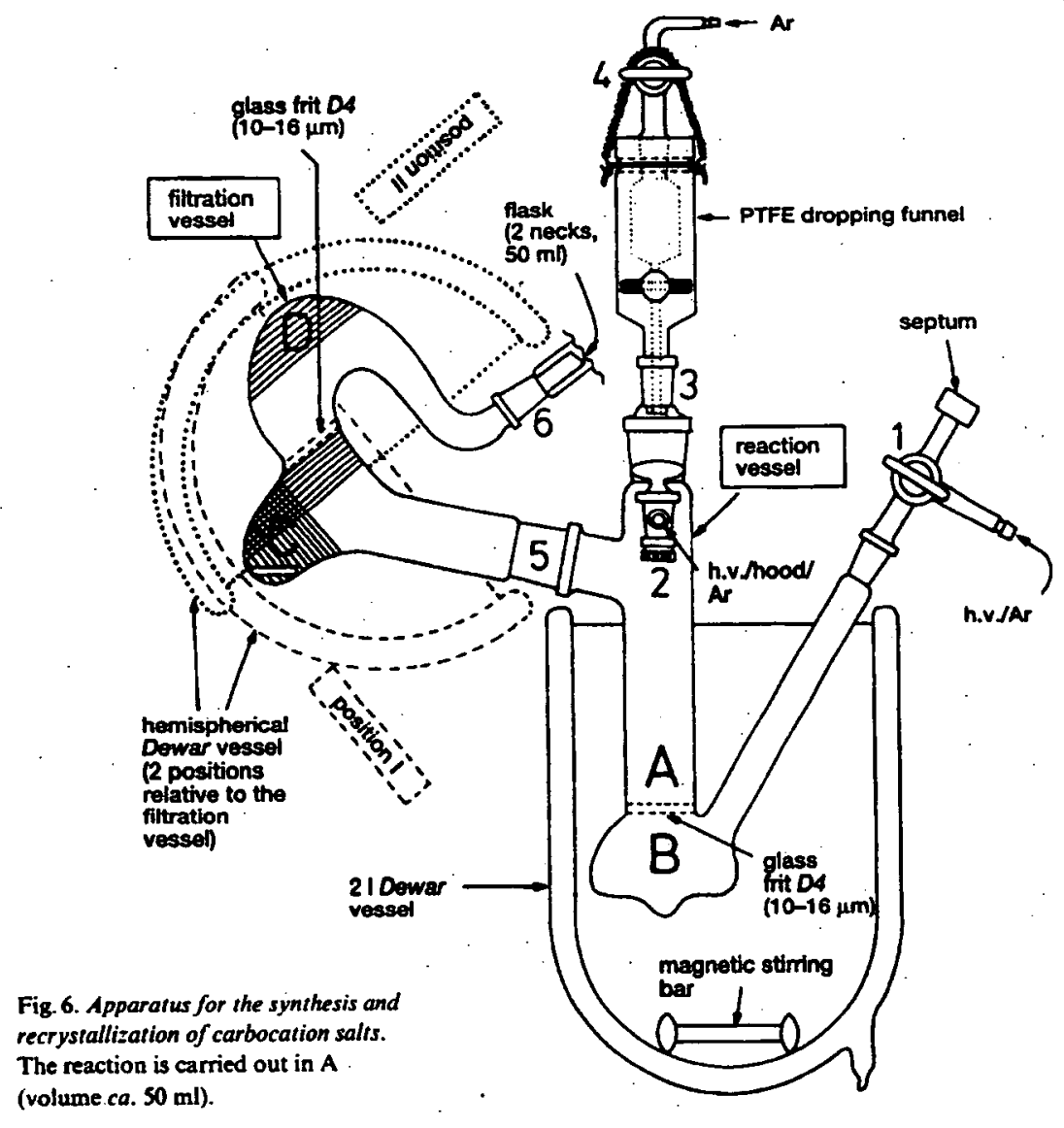

Fig. 6. Apparatus for the synthesis and recrystallization of carbocation salts. The reaction is carried out in $A$ (volume.ca. $50 \mathrm{ml}$ ).

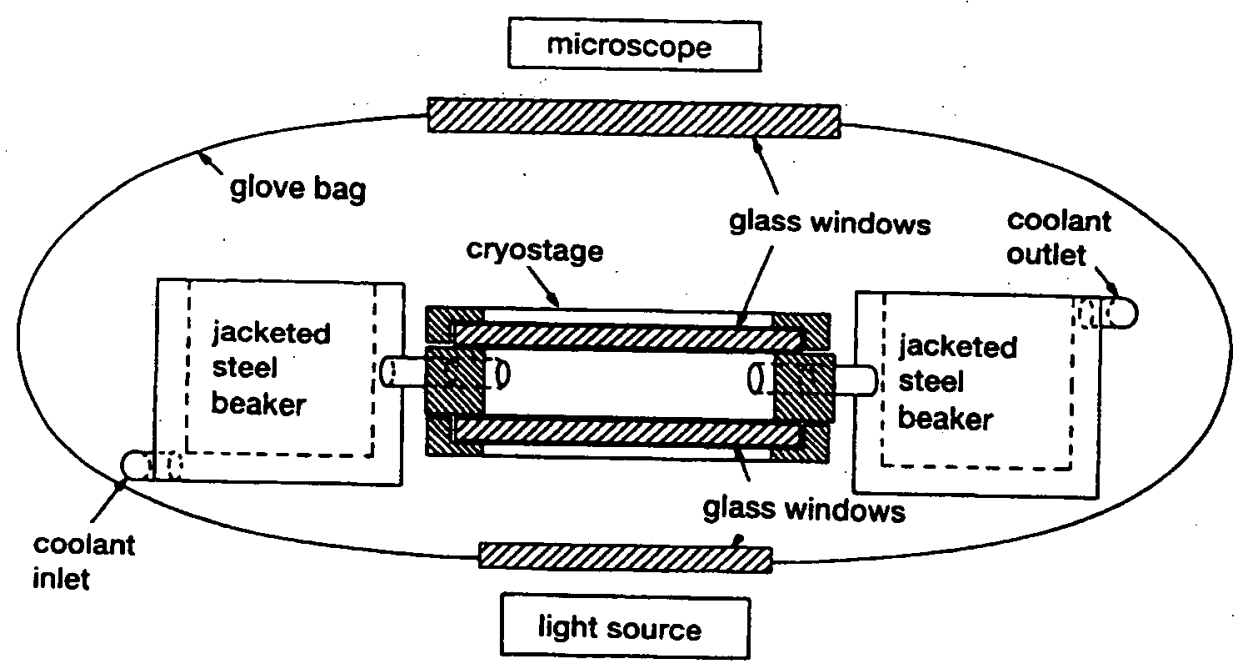

Fig. 7. Schematic drawing of ihe cryoslage. The whole stage with the beakers is made from stainless steel (welded: gas-tight) and cooled with circulating $\mathrm{MeOH}$ (cryostat). The stage is situated between two glass windows (in a metal frame) in a medium-sized glove bag. The $\mathrm{N}_{2}$ in the glove bag is circulating through a lower with molecular sieve as drying agent. All sealings of the glove bag (glass windows, coolant inlet and outlet. drying tower) are gas-tight. The working area on the cooled glass window of the cryostage has a diameter of $10 \mathrm{~cm}$. The metal parts of the cryostage are thermally insulated.

Sketch of the apparatus used for the preparation of crystalline Me-1 $\mathbf{1}^{+} \mathrm{Sb}_{2} \mathrm{~F}_{11}^{-}$(Laube, T. Helv. Chim. Acta 1994, 77, 943). 

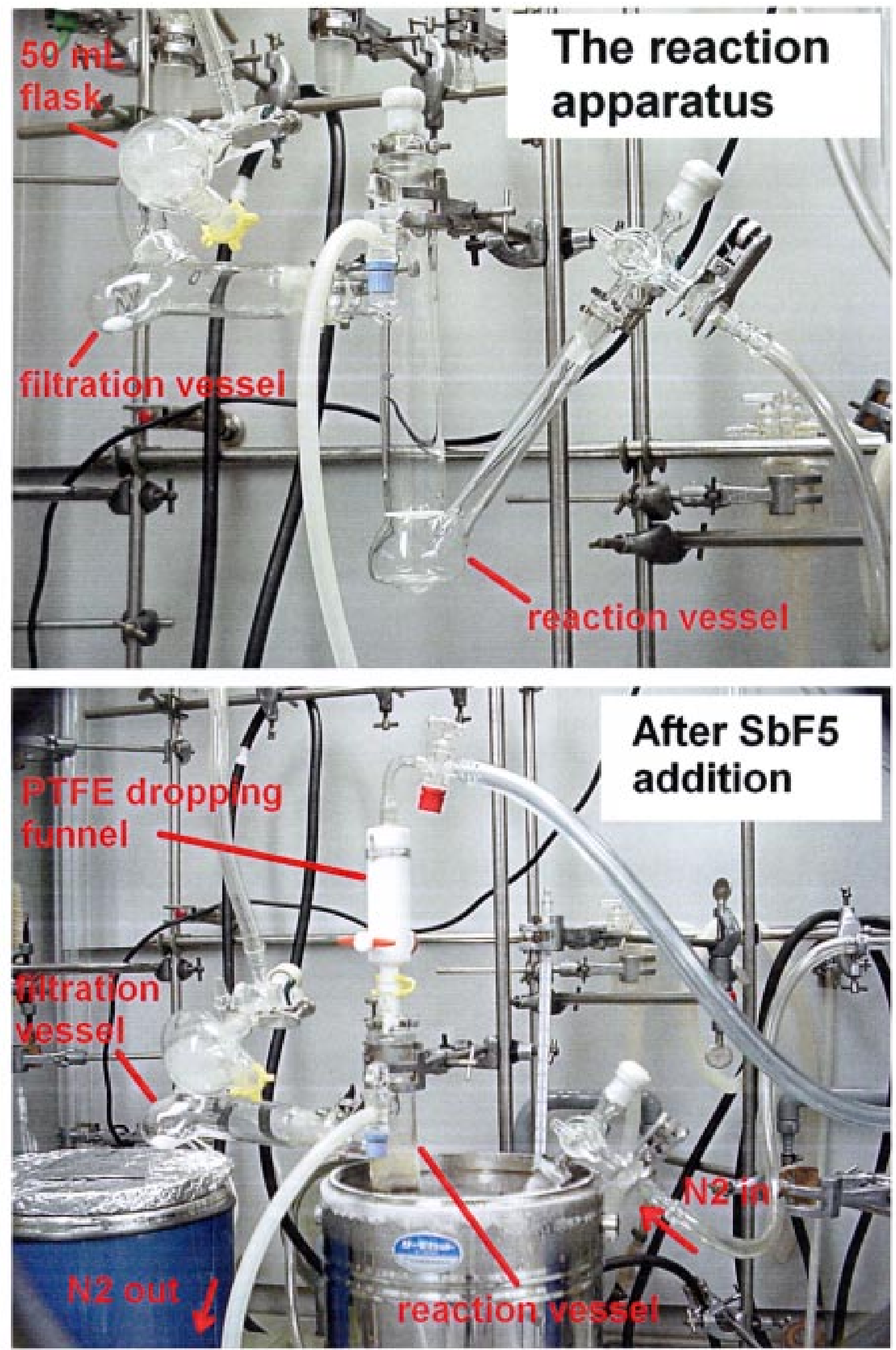
X-ray Crystal Structures of a Benzonorbornenyl Cation and of a Protonated Benzonorbornenol Thomas Laube J. Am. Chem. Soc.
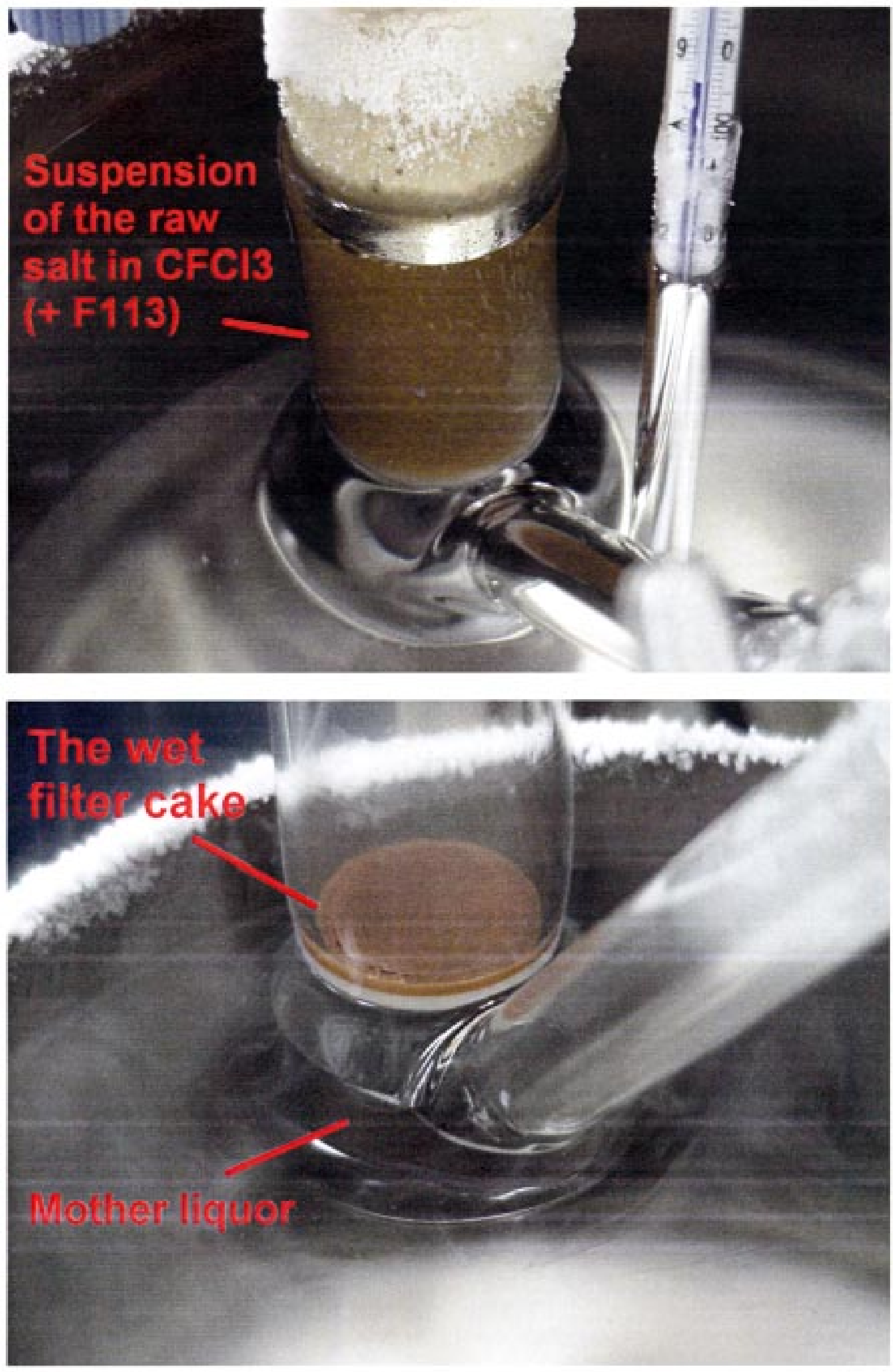

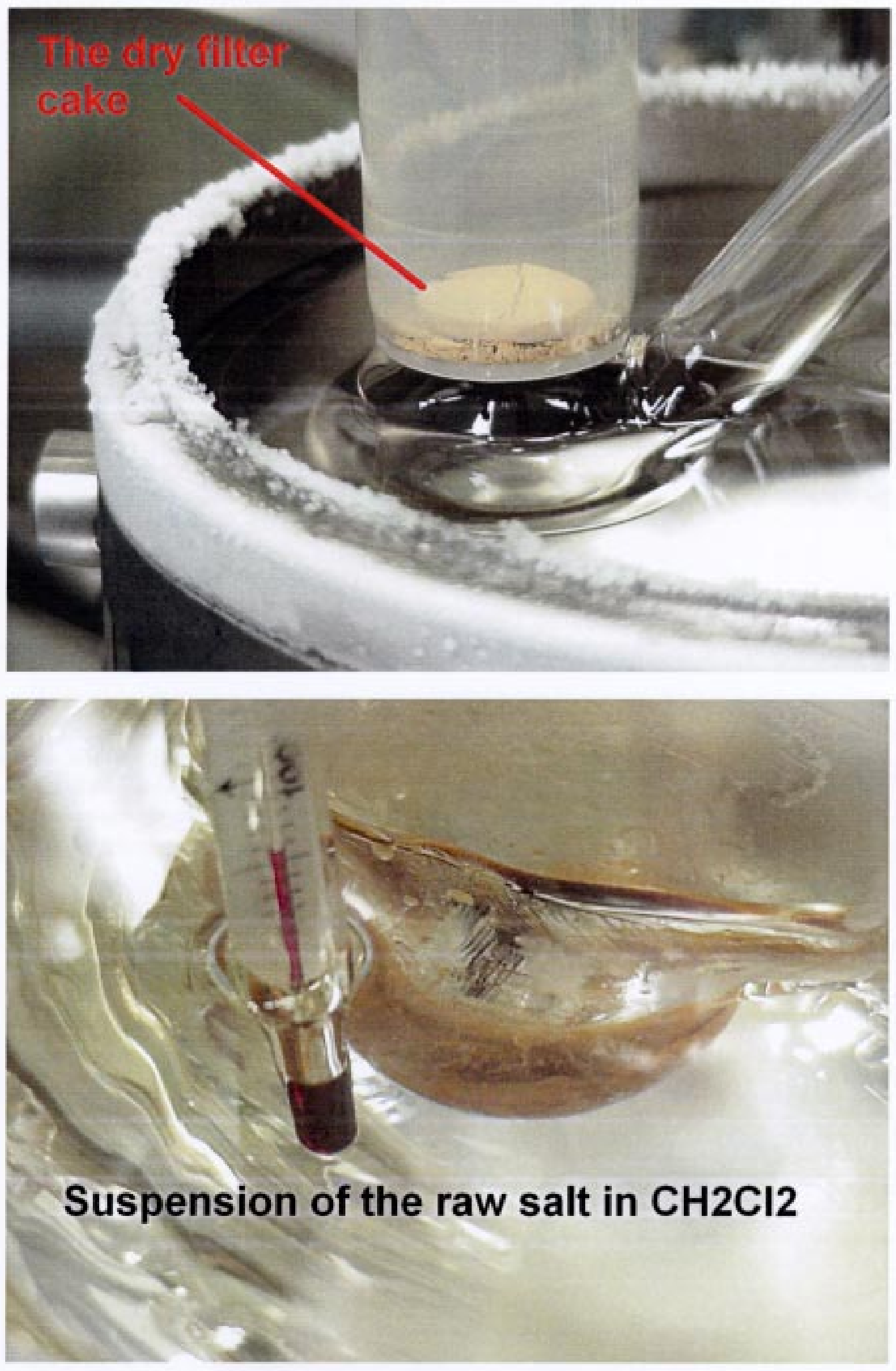
X-ray Crystal Structures of a Benzonorbornenyl Cation and of a Protonated Benzonorbornenol Thomas Laube

J. Am. Chem. Soc.

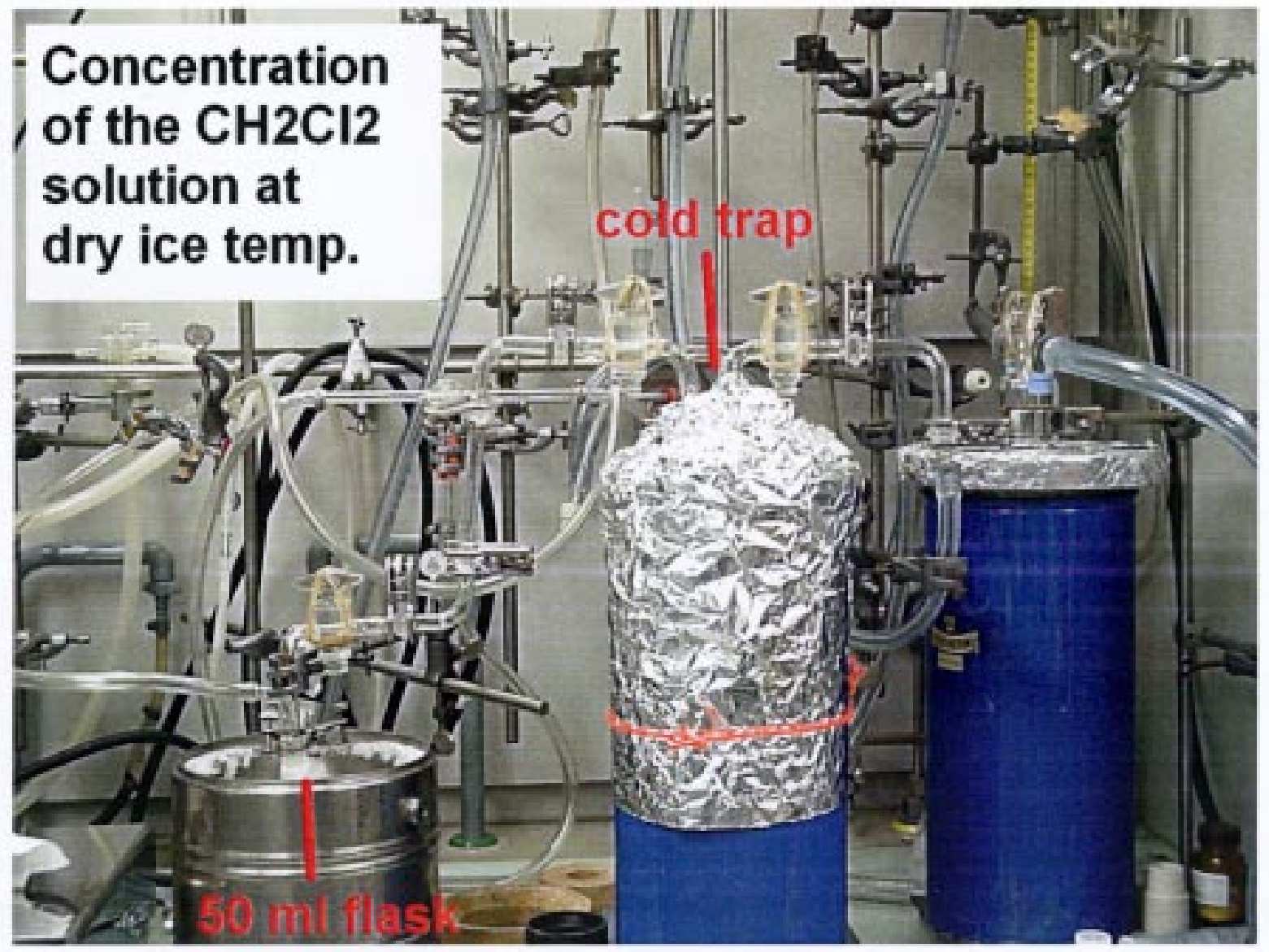

\section{After complete evaporation of the $\mathrm{CH} 2 \mathrm{Cl} 2$}

some small

crystals 
X-ray Crystal Structures of a Benzonorbornenyl Cation and of a Protonated Benzonorbornenol Thomas Laube J. Am. Chem. Soc.

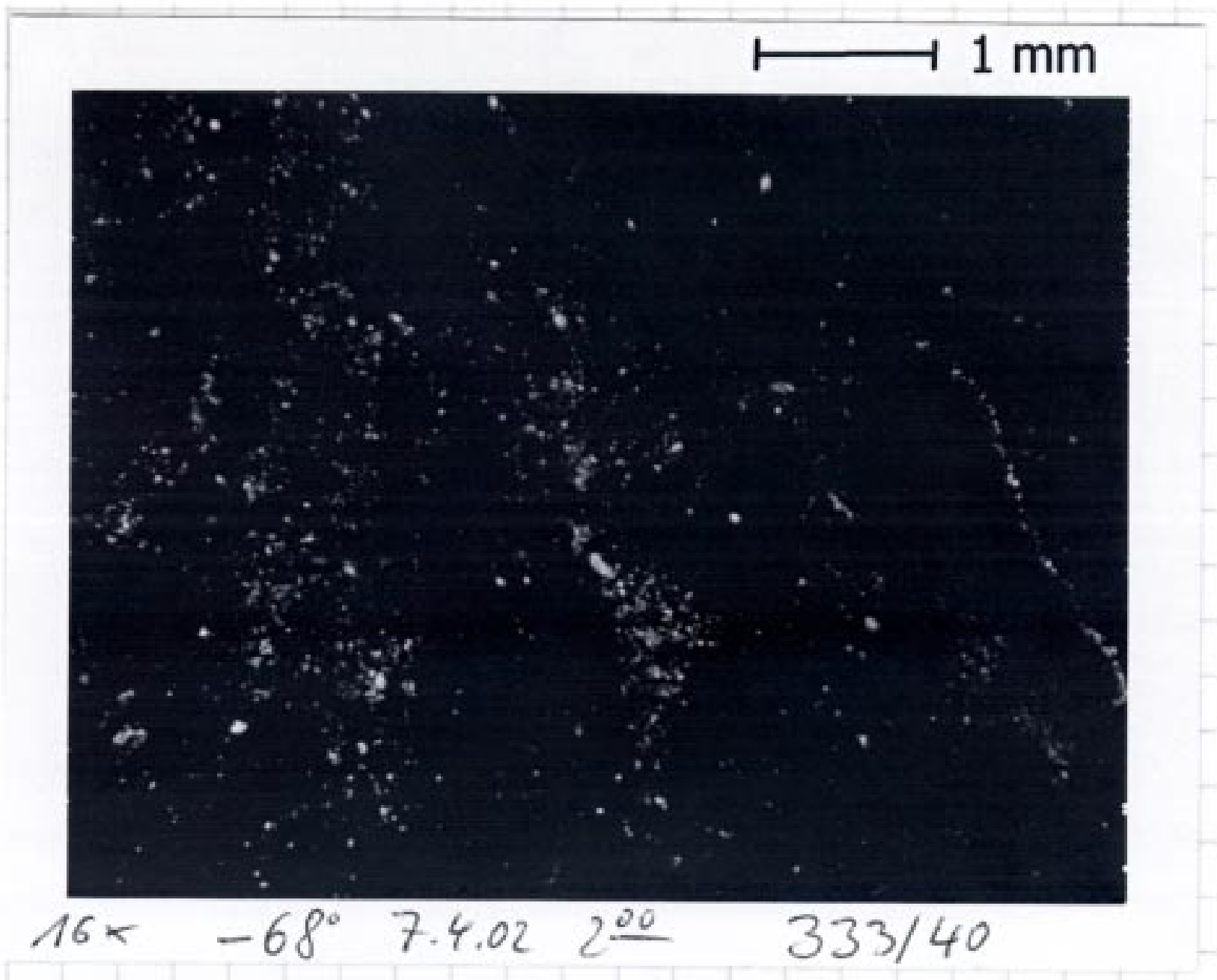

Crystals of Me-1 ${ }^{+} \mathrm{Sb}_{2} \mathrm{~F}_{11}{ }^{-}$under polarized light (crossed polarizers) at $-68 \mathrm{deg} \mathrm{C}$. Most crystals are very small $(<0.1 \mathrm{~mm})$. 
-(C) 2004 American Chemical Society, J. Am. Chem. Soc., Laube ja040115t Supporting Info Page 36 Thomas Laube J.Am. Chem. Soc.

\section{Crystal structure analysis of $5^{+} \mathrm{SO}_{3} \mathrm{Cl}^{-} \cdot \mathrm{CH}_{2} \mathrm{Cl}_{2}$}

Table 1. Crystal data and structure refinement for $5^{+} \mathrm{SO}_{3} \mathrm{Cl}^{-} \cdot \mathrm{CH}_{2} \mathrm{Cl}_{2}$.

\begin{tabular}{|c|c|}
\hline Identification code & c10301 \\
\hline Empirical formula & $\mathrm{C} 12 \mathrm{H} 15 \mathrm{C} 13 \mathrm{O} 4 \mathrm{~S}$ \\
\hline Formula weight & 361.65 \\
\hline Temperature & $160(2) \mathrm{K}$ \\
\hline Wavelength & $0.71073 \AA$ \\
\hline Crystal system, space group & monoclinic, $P Z_{1} / n$ \\
\hline Unit cell dimensions & $a=10.97570(10) \AA \quad \alpha=90^{\circ}$ \\
\hline & $b=6.17250(10) \AA$ \\
\hline & $c=23.3650(3) \AA$ \\
\hline Vol ume & $1540.17(3) \AA^{3}$ \\
\hline$Z$, Calculated density & 4, $1.560 \mathrm{Mg} / \mathrm{m}^{3}$ \\
\hline Absorption coefficient & $0.738 \mathrm{~mm}^{-1}$ \\
\hline$F(000)$ & 744 \\
\hline Crystal size & $0.3 \times 0.2 \times 0.2 \mathrm{~mm}$ \\
\hline Theta range for data collection & 2.29 to $30.02^{\circ}$. \\
\hline Limiting indices & $-15 \leq h \leq 15, \quad-8 \leq k \leq 8, \quad-32 \leq 7 \leq 32$ \\
\hline Reflections collected / unique & $37892 . / 4480[R($ int $)=0.0780]$ \\
\hline Completeness to theta $=30.02$ & $99.4 \%$ \\
\hline Refinement method & Fu11-matrix 1east-squares on $P$ \\
\hline Data / restraints / parameters & $4480 / 0 / 245$ \\
\hline Goodness-of-fit on $P$. & 1.025 \\
\hline Final $R$ indices $\left[\begin{array}{ll}I>2 & \sigma_{I}\end{array}\right]$ & $R 1=0.0392, \quad w R 2=0.1004$ \\
\hline$R$ indices (all data) & $R 1=0.0518, \quad w R 2=0.1073$ \\
\hline Largest diff. peak and hole & 0.350 and -0.563 e $\AA^{-3}$ \\
\hline
\end{tabular}


-(C) 2004 American Chemical Society, J. Am. Chem. Soc., Laube ja040115t Supporting Info Page 37 Thomas Laube J. Am. Chem. Soc.

Table 2a. Atomic coordinates and (equivalent) isotropic displacement parameters $\left(\AA^{2}\right)$ for $5^{+}$ $\mathrm{SO}_{3} \mathrm{Cl}^{-} \cdot \mathrm{CH}_{2} \mathrm{Cl}_{2}$. $U_{\mathrm{eq}}$ is defined as one third of the trace of the orthogonalized $U_{i j}$ tensor. For $\mathrm{H}$ atoms, $U_{\text {iso }}$ is given. The site occupation factors (sof) should be divided by the symmetry multiplicity (sm) for obtaining the correct content of the asymmetric unit.

\begin{tabular}{|c|c|c|c|c|c|c|}
\hline Atom & $x$ & $y$ & $z$ & $U_{e q}$ or $U_{i s o}$ & sof & $s m$ \\
\hline C1S & $0.8947(2)$ & $0.7177(4)$ & $0.03292(10)$ & $0.0423(4)$ & 1 & 1 \\
\hline Cl1s & $0.83572(5)$ & $0.98190(9)$ & $0.02261(3)$ & $0.05214(16)$ & 1 & 1 \\
\hline $\mathrm{C} 12 \mathrm{~S}$ & $0.78235(7)$ & $0.52680(10)$ & $-0.00137(3)$ & $0.05833(17)$ & 1 & 1 \\
\hline H1S & $0.961(2)$ & $0.715(4)$ & $0.0153(11)$ & $0.050(7)$ & 1 & 1 \\
\hline $\mathrm{H} 2 \mathrm{~S}$ & $0.921(3)$ & $0.696(5)$ & $0.0727(14)$ & $0.071(9)$ & 1 & 1 \\
\hline S & $0.20144(3)$ & $0.41277(7)$ & $0.178350(17)$ & $0.02479(10)$ & 1 & 1 \\
\hline $\mathrm{Cl}$ & $0.06835(4)$ & $0.23076(8)$ & $0.12280(2)$ & $0.03976(13)$ & 1 & 1 \\
\hline 01 & $0.18439(12)$ & $0.6258(2)$ & $0.15204(6)$ & $0.0330(3)$ & 1 & 1 \\
\hline H10B & 0.2459 & 0.6558 & 0.1377 & $0.042(9)$ & $0.24(4)$ & 1 \\
\hline 02 & $0.31813(11)$ & $0.3112(2)$ & $0.17365(6)$ & $0.0331(3)$ & 1 & 1 \\
\hline $\mathrm{H} 20 \mathrm{~B}$ & 0.3025 & 0.1965 & 0.1540 & $0.030(8)$ & $0.29(4)$ & 1 \\
\hline 03 & $0.17103(13)$ & $0.3948(2)$ & $0.23398(6)$ & $0.0411(3)$ & 1 & 1 \\
\hline C1 & $0.55837(14)$ & $0.0443(3)$ & $0.15542(7)$ & $0.0235(3)$ & 1 & 1 \\
\hline C2 & $0.52495(16)$ & $0.0724(3)$ & $0.08739(7)$ & $0.0265(3)$ & 1 & 1 \\
\hline C3 & $0.49575(16)$ & $-0.1621(3)$ & $0.06421(7)$ & $0.0281(3)$ & 1 & 1 \\
\hline $\mathrm{C} 4$ & $0.51502(15)$ & $-0.2997(3)$ & $0.12135(7)$ & $0.0250(3)$ & 1 & 1 \\
\hline $\mathrm{C} 4 \mathrm{~A}$ & $0.65398(15)$ & $-0.2915(3)$ & $0.14989(7)$ & $0.0244(3)$ & 1 & 1 \\
\hline C5 & $0.74803(16)$ & $-0.4437(3)$ & $0.15537(8)$ & $0.0308(4)$ & 1 & 1 \\
\hline C6 & $0.86909(16)$ & $-0.3827(3)$ & $0.18321(8)$ & $0.0346(4)$ & 1 & 1 \\
\hline C7 & $0.89575(16)$ & $-0.1738(3)$ & $0.20447(8)$ & $0.0347(4)$ & 1 & 1 \\
\hline $\mathrm{C} 8$ & $0.80105(16)$ & $-0.0192(3)$ & $0.19869(7)$ & $0.0303(3)$ & 1 & 1 \\
\hline $\mathrm{C} 8 \mathrm{~A}$ & $0.68013(14)$ & $-0.0808(3)$ & $0.17112(6)$ & $0.0238(3)$ & 1 & 1 \\
\hline $\mathrm{Cg}$ & $0.47191(14)$ & $-0.1414(3)$ & $0.16332(7)$ & $0.0237(3)$ & 1 & 1 \\
\hline 0 & $0.33920(11)$ & $-0.0791(2)$ & $0.14097(5)$ & $0.0273(2)$ & 1 & 1 \\
\hline $\mathrm{H} 1$ & $0.5534(18)$ & $0.171(3)$ & $0.1749(9)$ & $0.027(5)$ & 1 & 1 \\
\hline$H 2 X$ & $0.449(2)$ & $0.164(4)$ & $0.0760(9)$ & $0.034(5)$ & 1 & 1 \\
\hline $\mathrm{H} 2 \mathrm{~N}$ & $0.5954(19)$ & $0.136(3)$ & $0.0739(9)$ & $0.031(5)$ & 1 & 1 \\
\hline$H 3 x$ & $0.410(2)$ & $-0.181(4)$ & $0.0438(9)$ & $0.034(5)$ & 1 & 1 \\
\hline H3N & $0.5485(19)$ & $-0.216(3)$ & $0.0419(9)$ & $0.033(5)$ & 1 & 1 \\
\hline H4 & $0.4807(18)$ & $-0.443(3)$ & $0.1188(9)$ & $0.027(5)$ & 1 & 1 \\
\hline H5 & $0.7318(19)$ & $-0.586(4)$ & $0.1392(9)$ & $0.033(5)$ & 1 & 1 \\
\hline H6 & $0.930(2)$ & $-0.480(4)$ & $0.1872(10)$ & $0.039(6)$ & 1 & 1 \\
\hline $\mathrm{H} 7$ & $0.981(2)$ & $-0.138(4)$ & $0.2229(9)$ & $0.034(5)$ & 1 & 1 \\
\hline H8 & $0.820(2)$ & $0.123(4)$ & $0.2125(9)$ & $0.037(6)$ & 1 & 1 \\
\hline $\mathrm{H} 9$ & $0.4794(16)$ & $-0.197(3)$ & $0.2041(8)$ & $0.020(4)$ & 1 & 1 \\
\hline $\mathrm{H} 20 \mathrm{~A}$ & $0.328(3)$ & $0.050(6)$ & $0.1536(13)$ & $0.030(8)$ & $0.71(4)$ & 1 \\
\hline $\mathrm{H} 10 \mathrm{~A}$ & $0.293(3)$ & $-0.172(6)$ & $0.1480(14)$ & $0.042(9)$ & $0.76(4)$ & 1 \\
\hline
\end{tabular}


(1) 2004 American Chemical Society J. Am. Chem. Soc., Laube ja040115t Supporting Info Page 38 Thomas Laube J. Am. Chem. Soc.

Table 2b. Anisotropic displacement parameters $\left(\AA^{2}\right)$ for $5^{+} \mathrm{SO}_{3} \mathrm{Cl}^{-} \cdot \mathrm{CH}_{2} \mathrm{Cl}_{2}$.

\begin{tabular}{lllllll}
\hline Atom & $U_{11}$ & $U_{22}$ & $U_{33}$ & $U_{23}$ & $U_{13}$ & $U_{12}$ \\
\hline C1S & $0.0393(11)$ & $0.0436(11)$ & $0.0435(11)$ & $0.0083(9)$ & $0.0086(9)$ & $0.0048(9)$ \\
C11S & $0.0408(3)$ & $0.0375(3)$ & $0.0732(4)$ & $-0.0098(2)$ & $0.0028(2)$ & $0.0025(2)$ \\
C12S & $0.0737(4)$ & $0.0386(3)$ & $0.0626(4)$ & $-0.0087(2)$ & $0.0156(3)$ & $-0.0084(3)$ \\
S & $0.02161(19)$ & $0.0262(2)$ & $0.02715(19)$ & $0.00014(14)$ & $0.00694(14)$ & $0.00244(14)$ \\
C1 & $0.0325(2)$ & $0.0363(2)$ & $0.0497(3)$ & $-0.0116(2)$ & $0.00787(19)$ & $-0.00694(17)$ \\
01 & $0.0282(6)$ & $0.0259(6)$ & $0.0430(7)$ & $0.0029(5)$ & $0.0040(5)$ & $0.0005(5)$ \\
C2 & $0.0230(6)$ & $0.0305(7)$ & $0.0476(7)$ & $0.0010(5)$ & $0.0122(5)$ & $0.0044(5)$ \\
03 & $0.0407(7)$ & $0.0560(9)$ & $0.0298(6)$ & $0.0033(6)$ & $0.0144(5)$ & $0.0057(6)$ \\
C1 & $0.0238(7)$ & $0.0246(7)$ & $0.0218(7)$ & $-0.0006(6)$ & $0.0046(5)$ & $0.0028(6)$ \\
C2 & $0.0257(8)$ & $0.0297(8)$ & $0.0238(7)$ & $0.0053(6)$ & $0.0052(6)$ & $0.0029(6)$ \\
C3 & $0.0255(8)$ & $0.0361(9)$ & $0.0219(7)$ & $-0.0014(6)$ & $0.0036(6)$ & $0.0019(6)$ \\
C4 & $0.0229(7)$ & $0.0244(8)$ & $0.0281(7)$ & $-0.0013(6)$ & $0.0063(6)$ & $0.0016(6)$ \\
C4A & $0.0229(7)$ & $0.0289(8)$ & $0.0225(7)$ & $0.0037(6)$ & $0.0078(5)$ & $0.0038(6)$ \\
C5 & $0.0312(9)$ & $0.0310(9)$ & $0.0325(8)$ & $0.0045(7)$ & $0.0120(7)$ & $0.0085(7)$ \\
C6 & $0.0239(8)$ & $0.0473(11)$ & $0.0346(9)$ & $0.0127(8)$ & $0.0109(7)$ & $0.0139(7)$ \\
C7 & $0.0210(8)$ & $0.0518(11)$ & $0.0298(8)$ & $0.0104(8)$ & $0.0027(6)$ & $0.0020(7)$ \\
C8 & $0.0277(8)$ & $0.0370(9)$ & $0.0249(7)$ & $0.0032(7)$ & $0.0034(6)$ & $-0.0001(7)$ \\
C8A & $0.0225(7)$ & $0.0295(8)$ & $0.0197(6)$ & $0.0032(6)$ & $0.0052(5)$ & $0.0028(6)$ \\
C9 & $0.0184(7)$ & $0.0290(8)$ & $0.0239(7)$ & $0.0037(6)$ & $0.0055(5)$ & $0.0044(6)$ \\
D & $0.0198(5)$ & $0.0308(7)$ & $0.0319(6)$ & $0.0033(5)$ & $0.0076(4)$ & $0.0038(5)$ \\
\hline
\end{tabular}

Table 2c. Principal mean square atomic displacements $U$

\begin{tabular}{ccccc}
\hline Atom & $U_{\max }$ & $U_{\operatorname{med}}$ & $U_{\min }$ & $U_{\max } / U_{\min }$ \\
\hline C1S & 0.0519 & 0.0413 & 0.0336 & 1.54 \\
C11S & 0.0820 & 0.0396 & 0.0348 & 2.36 \\
C12S & 0.0756 & 0.0645 & 0.0349 & 2.17 \\
S & 0.0276 & 0.0268 & 0.0199 & 1.39 \\
C1 & 0.0565 & 0.0373 & 0.0255 & 2.22 \\
01 & 0.0458 & 0.0281 & 0.0252 & 1.82 \\
02 & 0.0478 & 0.0321 & 0.0194 & 2.46 \\
03 & 0.0583 & 0.0401 & 0.0250 & 2.33 \\
C1 & 0.0275 & 0.0217 & 0.0213 & 1.29 \\
C2 & 0.0331 & 0.0259 & 0.0204 & 1.62 \\
C3 & 0.0368 & 0.0261 & 0.0214 & 1.72 \\
C4 & 0.0286 & 0.0252 & 0.0213 & 1.34 \\
C4A & 0.0321 & 0.0216 & 0.0194 & 1.65 \\
C5 & 0.0420 & 0.0282 & 0.0223 & 1.88 \\
C6 & 0.0585 & 0.0279 & 0.0173 & 3.38 \\
C7 & 0.0560 & 0.0282 & 0.0199 & 2.81 \\
C8 & 0.0381 & 0.0296 & 0.0233 & 1.64 \\
C8A & 0.0310 & 0.0218 & 0.0187 & 1.66 \\
C9 & 0.0318 & 0.0225 & 0.0168 & 1.89 \\
0 & 0.0350 & 0.0286 & 0.0182 & 1.92 \\
\hline
\end{tabular}


(C) 2004 American Chemical Society, J. Am. Chem. Soc., Laube ja040115t Supporting Info Page 39 X-ray urystal oructuies UI d Thomas Laube J. Am. Chem. Soc.

Table 3. Bond lengths $(\AA)$ and angles (deg) for $5^{+} \mathrm{SO}_{3} \mathrm{Cl}^{-} \cdot \mathrm{CH}_{2} \mathrm{Cl}_{2}$.

\begin{tabular}{cc|cc}
$\mathrm{C}(1 \mathrm{~S})-\mathrm{Cl}(1 \mathrm{~S})$ & $1.751(2)$ & $\mathrm{C}(4)-\mathrm{C}(4 \mathrm{~A})$ & $1.518(2)$ \\
$\mathrm{C}(1 \mathrm{~S})-\mathrm{Cl}(2 \mathrm{~S})$ & $1.759(2)$ & $\mathrm{C}(4)-\mathrm{C}(9)$ & $1.534(2)$ \\
$\mathrm{C}(1 \mathrm{~S})-\mathrm{H}(1 \mathrm{~S})$ & $0.91(3)$ & $\mathrm{C}(4)-\mathrm{H}(4)$ & $0.96(2)$ \\
$\mathrm{C}(1 \mathrm{~S})-\mathrm{H}(2 \mathrm{~S})$ & $0.92(3)$ & $\mathrm{C}(4 \mathrm{~A})-\mathrm{C}(5)$ & $1.380(2)$ \\
$\mathrm{S}-\mathrm{O}(3)$ & $1.4190(13)$ & $\mathrm{C}(4 \mathrm{~A})-\mathrm{C}(8 \mathrm{~A})$ & $1.397(2)$ \\
$\mathrm{S}-\mathrm{O}(1)$ & $1.4450(13)$ & $\mathrm{C}(5)-\mathrm{C}(6)$ & $1.390(3)$ \\
$\mathrm{S}-\mathrm{O}(2)$ & $1.4527(12)$ & $\mathrm{C}(5)-\mathrm{H}(5)$ & $0.95(2)$ \\
$\mathrm{S}-\mathrm{Cl}$ & $2.0506(6)$ & $\mathrm{C}(6)-\mathrm{C}(7)$ & $1.389(3)$ \\
$\mathrm{O}(1)-\mathrm{H}(1 \mathrm{OB})$ & 0.84 & $\mathrm{C}(6)-\mathrm{H}(6)$ & $0.88(2)$ \\
$\mathrm{O}(2)-\mathrm{H}(2 \mathrm{OB})$ & 0.84 & $\mathrm{C}(7)-\mathrm{C}(8)$ & $1.394(3)$ \\
$\mathrm{C}(1)-\mathrm{C}(8 \mathrm{~A})$ & $1.514(2)$ & $\mathrm{C}(7)-\mathrm{H}(7)$ & $0.96(2)$ \\
$\mathrm{C}(1)-\mathrm{C}(9)$ & $1.526(2)$ & $\mathrm{C}(8)-\mathrm{C}(8 \mathrm{~A})$ & $1.388(2)$ \\
$\mathrm{C}(1)-\mathrm{C}(2)$ & $1.556(2)$ & $\mathrm{C}(8)-\mathrm{H}(8)$ & $0.94(2)$ \\
$\mathrm{C}(1)-\mathrm{H}(1)$ & $0.91(2)$ & $\mathrm{C}(9)-\mathrm{O}$ & $1.4803(18)$ \\
$\mathrm{C}(2)-\mathrm{C}(3)$ & $1.552(2)$ & $\mathrm{C}(9)-\mathrm{H}(9)$ & $0.997(18)$ \\
$\mathrm{C}(2)-\mathrm{H}(2 \mathrm{X})$ & $0.99(2)$ & $\mathrm{O}-\mathrm{H}(2 \mathrm{OA})$ & $0.87(3)$ \\
$\mathrm{C}(2)-\mathrm{H}(2 \mathrm{~N})$ & $0.98(2)$ & $\mathrm{O}-\mathrm{H}(1 \mathrm{OA})$ & $0.80(4)$ \\
$\mathrm{C}(3)-\mathrm{C}(4)$ & $1.555(2)$ & &
\end{tabular}

$\begin{array}{cc}\mathrm{Cl}(1 S)-\mathrm{C}(1 \mathrm{~S})-\mathrm{Cl}(2 S) & 111.32(12) \\ \mathrm{Cl}(1 \mathrm{~S})-\mathrm{C}(1 \mathrm{~S})-\mathrm{H}(1 \mathrm{~S}) & 105.0(16) \\ \mathrm{Cl}(2 \mathrm{~S})-\mathrm{C}(1 \mathrm{~S})-\mathrm{H}(1 \mathrm{~S}) & 109.3(16) \\ \mathrm{Cl}(1 \mathrm{~S})-\mathrm{C}(1 \mathrm{~S})-\mathrm{H}(2 \mathrm{~S}) & 107(2) \\ \mathrm{Cl}(2 \mathrm{~S})-\mathrm{C}(1 \mathrm{~S})-\mathrm{H}(2 \mathrm{~S}) & 112.8(19) \\ \mathrm{H}(1 \mathrm{~S})-\mathrm{C}(1 \mathrm{~S})-\mathrm{H}(2 \mathrm{~S}) & 111(2) \\ \mathrm{O}(3)-\mathrm{S}-\mathrm{O}(1) & 115.22(9) \\ \mathrm{O}(3)-\mathrm{S}-\mathrm{O}(2) & 115.69(8) \\ \mathrm{O}(1)-\mathrm{S}-\mathrm{O}(2) & 112.97(8) \\ \mathrm{O}(3)-\mathrm{S}-\mathrm{Cl} & 104.66(7) \\ \mathrm{O}(1)-\mathrm{S}-\mathrm{Cl} & 103.05(6) \\ \mathrm{O}(2)-\mathrm{S}-\mathrm{Cl} & 103.14(6) \\ \mathrm{S}-\mathrm{O}(1)-\mathrm{H}(1 \mathrm{OB}) & 109.5 \\ \mathrm{~S}-\mathrm{O}(2)-\mathrm{H}(2 \mathrm{OB}) & 109.5 \\ \mathrm{C}(8 \mathrm{~A})-\mathrm{C}(1)-\mathrm{C}(9) & 97.24(12) \\ \mathrm{C}(8 \mathrm{~A})-\mathrm{C}(1)-\mathrm{C}(2) & 106.91(12) \\ \mathrm{C}(9)-\mathrm{C}(1)-\mathrm{C}(2) & 101.56(13) \\ \mathrm{C}(8 \mathrm{~A})-\mathrm{C}(1)-\mathrm{H}(1) & 117.9(12) \\ \mathrm{C}(9)-\mathrm{C}(1)-\mathrm{H}(1) & 118.2(12) \\ \mathrm{C}(2)-\mathrm{C}(1)-\mathrm{H}(1) & 112.8(12) \\ \mathrm{C}(3)-\mathrm{C}(2)-\mathrm{C}(1) & 103.52(13) \\ \mathrm{C}(3)-\mathrm{C}(2)-\mathrm{H}(2 \mathrm{X}) & 110.2(13) \\ \mathrm{C}(1)-\mathrm{C}(2)-\mathrm{H}(2 \mathrm{X}) & 108.8(12) \\ \mathrm{C}(3)-\mathrm{C}(2)-\mathrm{H}(2 \mathrm{~N}) & 112.1(12) \\ \mathrm{C}(1)-\mathrm{C}(2)-\mathrm{H}(2 \mathrm{~N}) & 110.7(12) \\ \mathrm{H}(2 \mathrm{X})-\mathrm{C}(2)-\mathrm{H}(2 \mathrm{~N}) & 111.2(17) \\ \mathrm{C}(2)-\mathrm{C}(3)-\mathrm{C}(4) & 103.50(13) \\ \mathrm{C}(2)-\mathrm{C}(3)-\mathrm{H}(3 \mathrm{X}) & 112.8(13) \\ & \end{array}$

\begin{tabular}{|c|c|}
\hline$C(4)-C(3)-H(3 X)$ & $106.4(13)$ \\
\hline$C(2)-C(3)-H(3 N)$ & $115.3(13)$ \\
\hline$C(4)-C(3)-H(3 N)$ & $107.9(13)$ \\
\hline$H(3 X)-C(3)-H(3 N)$ & $110.2(18)$ \\
\hline$C(4 A)-C(4)-C(9)$ & $97.17(12)$ \\
\hline$C(4 A)-C(4)-C(3)$ & $106.42(13)$ \\
\hline$C(9)-C(4)-C(3)$ & $101.54(13)$ \\
\hline$C(4 A)-C(4)-H(4)$ & $113.5(12)$ \\
\hline$C(9)-C(4)-H(4)$ & $117.0(12)$ \\
\hline$C(3)-C(4)-H(4)$ & $118.5(12)$ \\
\hline$C(5)-C(4 A)-C(8 A)$ & $120.84(15)$ \\
\hline$C(5)-C(4 A)-C(4)$ & $132.25(16)$ \\
\hline$C(8 A)-C(4 A)-C(4)$ & $106.87(13)$ \\
\hline$C(4 A)-C(5)-C(6)$ & $118.24(17)$ \\
\hline$C(4 A)-C(5)-H(5)$ & $121.4(13)$ \\
\hline$C(6)-C(5)-H(5)$ & $120.3(13)$ \\
\hline$C(7)-C(6)-C(5)$ & $121.23(16)$ \\
\hline$C(7)-C(6)-H(6)$ & $120.0(15)$ \\
\hline$C(5)-C(6)-H(6)$ & $118.8(15)$ \\
\hline$C(6)-C(7)-C(8)$ & $120.66(16)$ \\
\hline$C(6)-C(7)-H(7)$ & $118.4(13)$ \\
\hline$C(8)-C(7)-H(7)$ & $121.0(13)$ \\
\hline$C(8 A)-C(8)-C(7)$ & $117.98(17)$ \\
\hline $\mathrm{C}(8 \mathrm{~A})-\mathrm{C}(8)-\mathrm{H}(8)$ & $121.7(13)$ \\
\hline$C(7)-C(8)-H(8)$ & $120.3(13)$ \\
\hline$C(8)-C(8 A)-C(4 A)$ & $121.04(15)$ \\
\hline$C(8)-C(8 A)-C(1)$ & $131.97(16)$ \\
\hline$C(4 A)-C(8 A)-C(1)$ & $106.95(13)$ \\
\hline
\end{tabular}


: (c) 2004 American Chemical Society, J. Am. Chem. Soc., Laube ja040115t Supporting Info Page 40 Thomas Laube J. Am. Chem. Soc.

\begin{tabular}{cc|cc}
$O-C(9)-C(1)$ & $110.52(13)$ & $C(4)-C(9)-H(9)$ & $115.0(11)$ \\
$O-C(9)-C(4)$ & $111.31(13)$ & $C(9)-O-H(2 O A)$ & $109.2(19)$ \\
$C(1)-C(9)-C(4)$ & $96.25(12)$ & $C(9)-O-H(1 O A)$ & $111(2)$ \\
$O-C(9)-H(9)$ & $106.5(10)$ & $H(2 O A)-O-H(10 A)$ & $116(3)$ \\
$C(1)-C(9)-H(9)$ & $117.2(11)$ & &
\end{tabular}

Table 4. Torsion angles (deg) for $5^{+} \mathrm{SO}_{3} \mathrm{Cl}^{-} \cdot \mathrm{CH}_{2} \mathrm{Cl}_{2}$.

$\begin{array}{cc}C(8 A)-C(1)-C(2)-C(3) & 67.25(15) \\ C(9)-C(1)-C(2)-C(3) & -34.08(15) \\ C(1)-C(2)-C(3)-C(4) & 0.19(15) \\ C(2)-C(3)-C(4)-C(4 A) & -67.56(15) \\ C(2)-C(3)-C(4)-C(9) & 33.56(15) \\ C(9)-C(4)-C(4 A)-C(5) & 148.30(18) \\ C(3)-C(4)-C(4 A)-C(5) & -107.4(2) \\ C(9)-C(4)-C(4 A)-C(8 A) & -34.02(15) \\ C(3)-C(4)-C(4 A)-C(8 A) & 70.30(15) \\ C(8 A)-C(4 A)-C(5)-C(6) & 0.8(2) \\ C(4)-C(4 A)-C(5)-C(6) & 178.21(16) \\ C(4 A)-C(5)-C(6)-C(7) & -0.7(3) \\ C(5)-C(6)-C(7)-C(8) & 0.4(3) \\ C(6)-C(7)-C(8)-C(8 A) & -0.1(3) \\ C(7)-C(8)-C(8 A)-C(4 A) & 0.1(2) \\ C(7)-C(8)-C(8 A)-C(1) & -177.15(16) \\ C(5)-C(4 A)-C(8 A)-C(8) & -0.5(2) \\ C(4)-C(4 A)-C(8 A)-C(8) & -178.51(14) \\ C(5)-C(4 A)-C(8 A)-C(1) & 177.37(14) \\ C(4)-C(4 A)-C(8 A)-C(1) & -0.63(16) \\ C(9)-C(1)-C(8 A)-C(8) & -147.21(17) \\ C(2)-C(1)-C(8 A)-C(8) & 108.33(19) \\ C(9)-C(1)-C(8 A)-C(4 A) & 35.23(15) \\ C(2)-C(1)-C(8 A)-C(4 A) & -69.23(16) \\ C(8 A)-C(1)-C(9)-O & -170.31(12) \\ C(2)-C(1)-C(9)-O & -61.33(15) \\ C(8 A)-C(1)-C(9)-C(4) & -54.75(13) \\ C(2)-C(1)-C(9)-C(4) & 54.24(13) \\ C(4 A)-C(4)-C(9)-O & 169.25(12) \\ C(3)-C(4)-C(9)-O & 60.79(16) \\ C(4 A)-C(4)-C(9)-C(1) & 54.32(13) \\ C(3)-C(4)-C(9)-C(1) & -54.13(13) \\ O(3)-S-O(1)-H(10 B) & 136.6 \\ O(3)-S-O(2)-H(2 O B) & 105.6 \\ & \\ C & \end{array}$

